\title{
A Scoping Review of Stress Measurements and Psychometry in Police Research
}

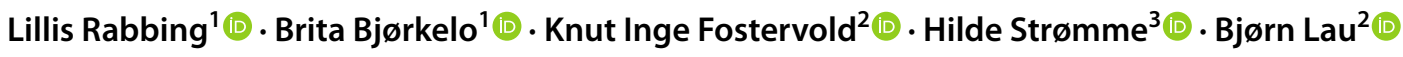

Accepted: 31 January 2022 / Published online: 23 February 2022

(c) The Author(s) 2022

\begin{abstract}
Despite a growing body of research, there is no systematic body of evidence that establishes the rigour of existing measures of stress among police. The aim of this scoping review was to investigate (1) the diversity of stress measures used in police research and (2) the psychometric properties of such measures and the ways in which they are utilised. The systematic literature search discovered 16,216 records, which were reduced to 442 records of relevance. A total of 20 qualitative and 422 quantitative studies were found to be relevant, including a total of 129 unique measures, of which the majority showed satisfactory reliability (Cronbach's alpha $\geq 0.80$ ). The identified measures pertain to four main categories: police-specific, perceived stress, psychological and physiological outcomes (including mood and affect changes), and assessment batteries. The measures have a general tendency to emphasise illness, and police-specific stressors pertain mostly to traditional police work. Measures should be chosen based on the aspect of the stress phenomenon that is to be investigated. This study provides detailed recommendations concerning how to use these measures to advance research concerning stress among police.
\end{abstract}

Keywords Stress $\cdot$ Police $\cdot$ Law enforcement $\cdot$ Scoping review $\cdot$ Stress measurement $\cdot$ Psychometric properties

Stress is ubiquitous in modern society, and few areas in contemporary psychology receive more attention (Hobfoll 1989; Robinson 2018; van Woerkom et al. 2021). The strong association between work-related stress and reduced wellbeing is well documented (Beehr and Newman 1978; Brown and Campbell 1994; Dewe and Cooper 2017; Johnson et al. 2005; Spielberger and Reheiser 1994). Working in the police service, either as a police officer (Abdollahi 2002; Brown and Campbell 1994; Purba and Demou 2019; Violanti et al. 2017) or as a civilian (Alderden and Skogan 2014; Ermasova et al. 2020), is no exception to this rule. The prevalence of stressrelated problems is higher among employees in the police service than among members of the general public (Morash et al. 2006; Slate et al. 2007; Violanti et al. 2013a, b). The recognition that policing is a hazardous and stressful profession

Lillis Rabbing

lillis.rabbing@phs.no

1 Norwegian Police University College, Post Box 2109, Vika, 0125 Oslo, Norway

2 Department of Psychology, University of Oslo, Forskningsveien 3A, 0373 Oslo, Norway

3 Library of Medicine and Science, University of Oslo, Sognsvannsveien 20, 0372 Oslo, Norway has stimulated a variety of studies focusing on the relationship between stress and pathology, which have been conducted with varying research designs and measurement methods (Abdollahi 2002; Queriós et al. 2020; Webster 2014).

Police work affects society and citizens as a whole (Cockcroft 2020; Symonds 1972; Wilson 1978) either with an emphasis on law enforcement or service provision. Many tasks performed by police are incident-driven, whether they are performed by a patrol unit, an investigation division, or by the administration. The tasks are also visible, often time limited, and have no simple consensus or known solution (Anshel et al. 1997; Hansson et al. 2017). Each case and situation must be addressed in accordance with the individuals and context involved (Hesketh and Cooper 2017) either by using analytical considerations or intuitively. Irrespective of policy and practice, any use of force must be necessary, proportionate, and in accordance with human rights and equalities legislation.

Stress responses affect cognitive and motor skills, e.g., physiological requirements needed for urgent situations (Anderson et al. 2019; Anshel et al. 1997; Regehr and LeBlanc 2017; Yaribeygi et al. 2017). Employees lacking the ability to cope may pose a threat to themselves, colleagues, and the public (Saunders et al. 2019; Webb 
and Smith 1980) and may impair the performance of law enforcement (Bitner 2019; Shane 2020). Hence, police work and the ways in which working conditions, stress, and strain influence different facets of policing have received considerable academic attention since the early 1970s (Stratton 1978). However, a systematic overview of the rigour and properties of existing measures of stress among police is missing. This scoping review fills this gap.

\section{Stress as a Phenomenon}

A common way to understand psychological stress is through the transactional model (Lazarus 1995), according to which cognition and subsequent emotions are important considerations in behavioural response to a stimulus (Robinson 2018). Despite disagreements concerning conceptual issues and definitions in stress research, there seems to be a consensus on the importance of individual differences in responses to stressors (Anshel et al. 1997). The transactional model "highlights the cognitive process of appraisal as a mediator when confronted with a stressor" (Robinson 2018, p. 8).

Understanding personal involvement and the particular context is crucial to ameliorate or prevent stress at the workplace (Lazarus 1995). Once the appraisal process begins, the degree to which an individual considers a situation to be a source of stress depends on the subjectively perceived balance among environmental demands, contextual constraints, and resources, as well as individual coping abilities (Adams and Mastracci 2020; Dewe 1991; Lazarus 1995; McEwen 2012; Shane 2020). The appraisal process integrates two sets of forces in this context, namely, the individual's agenda and the environmental realities affecting the outcome (Lazarus 1995). Primary appraisals refer to whether the individual has a personal stake in the encounter. Secondary appraisals concern coping options based on available social and organisational resources and the amount of control that individuals feel they have over the situation. The appraisal processes and subsequent reappraisals are governed by the cumulative sum of contextual, situational, or private disturbances (Evans and Coman 1993; Gächter et al. 2011) and are a function of demands, available internal or external resources, and support (Rodrigues et al. 2019). Thus, the same situation or difficulty may be interpreted as harmless by one individual and as stressful by another (McEwen and Sapolsky 1995; Yaribeygi et al. 2017). This fact implies that situations, chronic working conditions, and daily hassles, whether acute or not, become stressful when appraised as such and not simply because they occur (Anshel 2000; Gudjonsson and Adlam 1985).

Untreated levels of stress, accompanied by loss of control and composure, cause impaired perception, memory, decision-making, reaction, and work performance (Arble et al. 2019; Gutshall et al. 2017; Larsson et al. 1988; McEwen and
Sapolsky 1995; Regehr and LeBlanc 2017; Shane 2020). On the other hand, mastery of stressful situations entails positive beliefs and emotions (Anshel et al. 1997; Faragher et al. 2004) and fosters concentration, skills, performance, and eagerness (Anshel et al. 1997; Nisar and Rasheed 2020; Noblet et al. 2012).

\section{Stress in the Police Context and How It Is Measured}

The various measures used to study stress among police are based on differing underlying theoretical assumptions and elements of the stress phenomenon (Dollard et al. 2013; Slate et al. 2007; Webster 2013). The measurements assess a variety of issues, such as adverse life events, stressful environments, the individual's physiological and psychological responses, and the interaction between the individual and the environment (Bhui et al. 2016). By measuring the presence and magnitude of occupational stressors, stress responses, and mood changes among police, one seeks to understand the work challenges, demands, and requirements involved in police work. The main idea is that this investigation may help to provide safe and engaging work conditions for police and to secure citizens' legal and human rights in the communities that police serve and protect.

One way of measuring stress is to inquire into policespecific stressors such as acute or chronic stressors, daily difficulties, or events of significance to policing. Another approach is to ask respondents to rate their general perception of stress. The latter approach contributes to the understanding of general and individual factors associated with perceived stress, which are not necessarily police-specific. A third way of measuring stress is to study the prevalence of reactions and outcomes, i.e., distress and strain, such as the impact on cognitive capacity, concentration, risk taking, withdrawal, anxiety, anger, irritability, and physical capacity or ailments.

Studies aimed at identifying and evaluating the general effect of occupational stress on physical and mental health, regardless of profession, seek to provide a comprehensive characterisation of work stressors in the work environment (Hurrell et al. 1998). Theoretical models have guided the construction of various generic measurements and batteries of scales. The aim of these models is to increase knowledge concerning how to foster practices that will either reduce job stress or improve an individual's adjustment to stress. Such batteries mainly contain three types of variables: work stressors, strain, and health outcomes. While comprehensive measurements may provide a more profound understanding, briefer measurements might represent an efficient first-pass psychosocial risk assessment for the identification of areas that warrant in-depth assessment and targeted risk reduction activities. 
The sources of stress in the policing profession are often divided into operational and organisational demands or stressors (Hillgren et al. 1976; Kroes 1976; Reiser 1976; Shane 2020; Symonds 1970; Violanti et al. 2017), with the latter identified as the predominant stress source (Shane 2020; Stinchcomb 2004; Violanti et al. 2017). The operational demands impinging police work comprise acute (e.g., critical on-the-spot decisions, responding to high-risk calls, high-speed pursuits, missing or nonfunctioning equipment), and chronic stressors (e.g., patrol activities, inactivity and boredom, traffic control activities, help and safety duties, and the ineffectiveness of the judicial or correctional systems). Although rarer in frequency (Berg et al. 2005; Hickman et al. 2011; Padilla 2020), traumatic events and threats to physical and psychological health are also included in the category of operational demands.

Organisational stress refers to chronic (Anshel et al. 1997; Dollard et al. 2003) exposure to exacerbating and enduring pressure (Bishopp et al. 2016), including stressors such as role conflicts and ambiguity, work overload, underutilisation of skills, lack of supervisor support, bureaucratic procedures, insufficient staffing, and record keeping (Berg et al. 2006; Kop et al. 1999; Shane 2013, 2020; Violanti et al. 2017). In addition, stressors from the sociopolitical context of policing (Demou et al. 2020; Saunders et al. 2019; Stratton 1978) are also involved. Sociopolitical pressure refers to the ways in which employees in the police are treated and trusted by the public, media, other organisations, or family and friends (Brown 2016; Can et al. 2015; Ermasova et al. 2020; Van Hasselt et al. 2003).

Cognitions and subsequent emotions are important considerations in behavioural responses to stimuli (Anshel et al. 1997; Robinson 2018). Serious physiological adverse health outcomes in policing include musculoskeletal disorders (Douma et al. 2018; Larsen et al. 2018), cardiovascular diseases, and metabolic syndrome (Violanti et al. 2018a, b; Yamauchi et al. 2018; Zimmerman 2012). Examples of psychological and behavioural adverse health outcomes reported by employees in the police service include burnout (Adams and Mastracci 2020; Kop et al. 1999; McCarty et al. 2019), depression, anxiety, suicide (Berg et al. 2003; Bishopp and Boots 2014; Violanti et al. 2009, 2019; Violanti et al. 2013a, b), sleep disorders (Hartley et al. 2014; Ma et al. 2019), and posttraumatic stress disorder (PTSD) (Lees et al. 2019; Lilly et al. 2009; Stephens and Long 1998; Violanti et al. 2018a, b).

\section{Psychometric Properties}

The psychometric properties of measurement instruments refer to their reliability and validity. The evaluation of psychometric properties tries to establish the scientific robustness of the scale and the assessments made (de Souza et al.
2017). As the phenomenon of stress cannot be directly observed or quantified through self-report measures, the methodological approach employs an indirect measurement design, i.e., a scale on which respondents rate their perceptions of stress-related sources or events. The indirect approach is a hypothetical construct in which a latent variable (of stress), composed of carefully evaluated and selected items, is measured.

Neither reliability nor validity refers to inherent properties of the measure but to interactions among the scales, the group being tested, and the conditions (Keszei et al. 2010). Reliability is a property of the measurement as a whole (Danner 2016) and indicates whether the measurement is consistently able to reproduce similar results across time and space (Keszei et al. 2010). Validity refers to the meaning and interpretation of the scores; do we measure what we intend to measure?

As measurements cannot be valid unless they are reliable (Keszei et al. 2010), reliability is a way of assessing the psychometric properties of measurements. Cronbach's alpha assesses the degree to which a scale or composite is consistent, whether internally or temporally, in measuring variation in a sample (Edwards et al. 2021) and represents one way, among others, to assess reliability. Known discussions of the limitations of the use of Cronbach's alpha compared to other methods are its assumptions of uncorrelated errors, tau equivalence, unidimensionality, and normality (TrizanoHermosilla and Alvarado 2016). Nevertheless, Cronbach's alpha is the most commonly used reliability index (McNeish 2018; Peterson 1994; Raykov and Marcoulides 2019). The expression of alpha, a number between 0 and 1 , indicates higher consistency with a higher score. Although universally accepted norms seemingly are lacking, Nunnally's (1978) recommendations that acceptable levels of reliability are $\geq 0.70, \geq 0.80$, and $\geq 0.90$ for preliminary, basic, and applied research, respectively, have been widely applied.

\section{Aims of the Study}

Despite a growing body of research into the concept and measurement of stress among police (Cohen et al. 2019; Webster 2014), there is no systematic body of evidence that establishes the rigour of existing stress measures and their psychometric properties. In a police setting, a lack of knowledge concerning the utility of existing stress measures hampers the development of the research field as well as the possibility of promoting wellbeing and preventing ill health (Slate et al. 2007). This study establishes the rigour of existing and applied measures of stress among police by conducting a scoping review that investigates (1) existing stress measurements in the context of the policing profession and (2) the psychometric properties of such measures. In this study, we employ an acceptable Cronbach's alpha score to 
express a measurement's ability to detect and systematically investigate the type and form of stress that it is assumed to measure when applied to the police.

\section{Method}

\section{Procedure}

Research into stress among police is characterised by a plethora of definitions, measurements, and methods. A scoping review approach allows openness to stress as a concept and to study designs (Tricco et al. 2018). As our aim is to map and summarise existing measurements of stress among police, the criteria for choosing a scoping review approach were fulfilled (Munn et al. 2018; Peters et al. 2020, 2015).

\section{Search Methods}

The first and second authors (LR and BB) planned the study and decided on search terms. The PICO approach (Schardt et al. 2007) was used in discussions with two librarians (HS, the fourth author, and ATK). Databases were chosen to investigate the literature (i.e., articles that we call records in the following) published in the fields of medicine, psychology, law enforcement, and science in general. HS and ATK planned and conducted searches from the inception of the project to June 2019 without any restrictions in MEDLINE (Ovid), Embase (Ovid), PsycINFO (Ovid), AMED (Ovid), The Cochrane Library (Wiley), CINAHL (EBSCO), Academic Search Premier (EBSCO), Criminal Justice Abstracts (EBSCO), International Security \& Counter Terrorism Reference Center (EBSCO), and Scopus. The searches pertained to subject headings (where applicable) and to words in the text concerning police and law enforcement, combined with terms for perceived stress, work-related stress, demands, requirements, or working conditions. For complete search strategies, see the Appendix. All search results were exported to EndNote, and duplicates were removed.

\section{Inclusion and exclusion criteria}

Criteria for inclusion were first and foremost that the record included a measurement or an inventory tool pertaining to stress that was applied in a police setting. Both qualitative and quantitative studies were included, as measurements may be applied in both. The inclusion and exclusion criteria are reported in Table 1.

\section{Screening Process}

The first two steps in the screening process used the internet and the mobile tool Rayyan (Ouzzani et al. 2016). In the screening process, we restricted inclusion to English or Nordic languages only. See Fig. 1 for a flow diagram describing the process based on the PRISMA procedure (Moher et al. 2009).

Rayyan was used by two reviewers to screen the same records independently before comparing the results. This procedure was used to ensure transparency, clarity, and traceability throughout the selection process. LR performed the initial screening process, assessing titles and abstracts. This process reduced the number of eligible records from 16,231 to 2191 . The second part was organised in five waves, which were completed in pairs by LR alongside either BB, KIF (the third author), or BL (the final author) using the blind mode of Rayyan. Between every wave and before starting the next wave, each pair completed dialogues to resolve conflicting decisions. This procedure contributed to the task of establishing interrater reliability for each pair and wave. The number of conflicting decisions decreased from 15 to $6 \%$. Records judged as possibly relevant based on titles and abstracts $(n=956)$ were read in full.

Table 1 Inclusion and exclusion criteria

\begin{tabular}{|c|c|}
\hline Inclusion & Exclusion \\
\hline $\begin{array}{l}\text { Sample: Police officers; police employees without } \\
\text { police education; staff employed in police or law } \\
\text { enforcement professions }\end{array}$ & Nonpolice personnel, recruits, students, or employees in nonpolice occupations \\
\hline $\begin{array}{l}\text { Phenomenon of interest: Studies subjectively } \\
\text { measuring work related stress among employees } \\
\text { in the police or law enforcement }\end{array}$ & $\begin{array}{l}\text { Studies in which stress was measured biometrically (blood pressure, heart rate, cortisol, } \\
\text { saliva, etc.), animal studies (police dogs, horses, ants, etc.), studies only concerning out- } \\
\text { comes (burnout, sleep deprivation, PTSD), coping, shift-work, job satisfaction, or quality } \\
\text { of life were also excluded if they did not include a measurement of subjective stress }\end{array}$ \\
\hline Type of publications: Peer-reviewed & $\begin{array}{l}\text { Reviews, nonpeer-reviewed publications (e.g., theses, dissertations, conference papers, } \\
\text { book sections), publications focusing on historical, ethical, legal, or other issues without } \\
\text { using a measurement of stress }\end{array}$ \\
\hline Design: Quantitative and qualitative studies & Qualitative studies in which there was no structure that allowed for a measurement of stress \\
\hline
\end{tabular}


Fig. 1 Summary of search, adapted from PRISMA
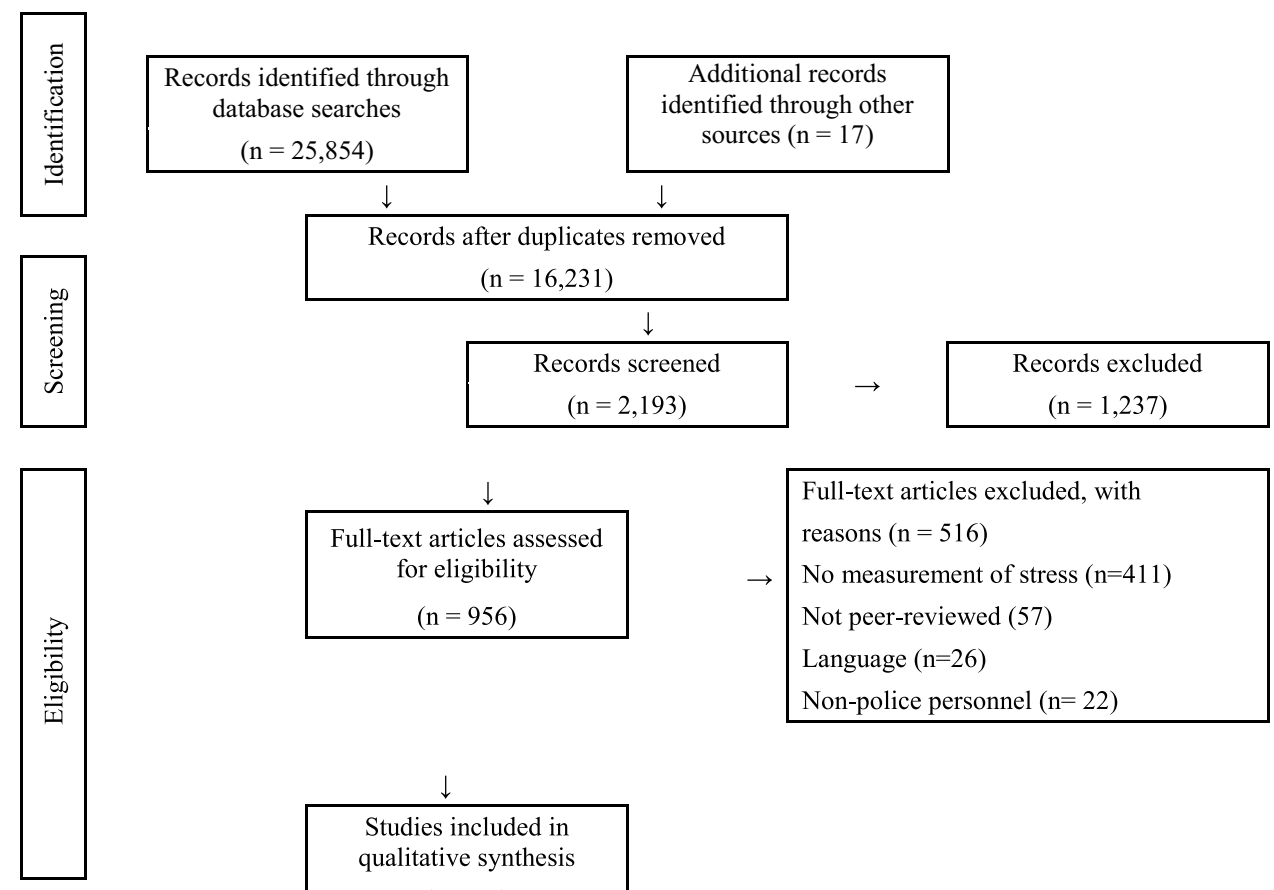

$$
\rightarrow \begin{aligned}
& \text { Full-text articles excluded, with } \\
& \text { reasons }(\mathrm{n}=516) \\
& \text { No measurement of stress }(\mathrm{n}=411) \\
& \text { Not peer-reviewed }(57) \\
& \text { Language }(\mathrm{n}=26) \\
& \text { Non-police personnel }(\mathrm{n}=22)
\end{aligned}
$$

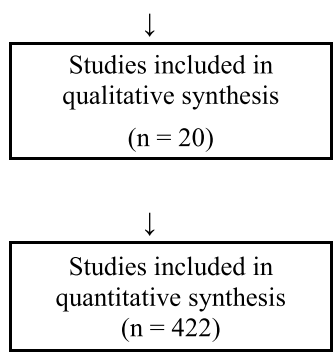

\section{Data Extraction}

Based on 956 records, a total of 20 qualitative studies and 422 quantitative studies were found to be relevant. From the included studies, measurements of subjective stress among employees in the police and the psychometric properties (i.e., Cronbach's alpha) of such measurements were extracted.

\section{Results}

This scoping review investigates (1) existing stress measurements in the police context and (2) the reliability of such measurements as measured by Cronbach's alpha.

The 20 qualitative studies provide insight into the early establishment and identification of the factors that contribute to police stress (Kroes et al. 1974a, b; Wexler and Logan 1983). However, the qualitative studies did not include measurements of stress in the form of closed questions or checklists and were therefore excluded from further analyses.

Based on 422 quantitative studies, we identified that 129 unique subjective measurements of stress had been used in police research. Within this group, 89 measurements reported psychometric properties (Cronbach's alpha), which ranged between 0.16 and 0.96 . A slight majority (54\%, $n=70$ ) of the measurements reported a Cronbach's alpha score over 0.80 , indicating satisfactory reliability among the police population.

The measures were divided into four major categories based on the ways in which they predominantly examine and target different aspects of the police stress phenomenon: police-specific (46 measurements, Table 2), perceived stress (23 measurements, Table 3), psychological and physiological outcomes as well as mood and affect (30 measurements, Table 4), and assessment batteries (30 measurements, Table 5). The four categories overlap in certain respects (e.g., common features concerning how stress is measured) and differ in terms of conceptual issues and national contexts of development and application. Most measures also seem to be combined when applied to the police. For example, police-specific measures are applied in combination with one or more measures from one of the other main categories.

The measurements are arranged chronologically based on when they were first developed. Information concerning at least one study in our material in which the measure was used, as well as information concerning any revised versions, are included. The tables list, from left to right, the name of the measurement, its abbreviation, the name of the developer(s), and the measurement's years of development 
Table 2 Police-specific work demand/stress measurements

\begin{tabular}{|c|c|c|c|c|c|c|}
\hline No & Name & Abbreviation & Author & Number of items & Response scale $(*)$ & Cronbach's alpha ( $a$ ) \\
\hline 1 & $\begin{array}{l}\text { Police Stress Survey } \\
\text { Modified Police Stress } \\
\text { Survey }\end{array}$ & PSS, MPSS & $\begin{array}{l}\text { Spielberger et al. 1981; White } \\
\text { et al. 1985; Patterson 1992; } \\
\text { Martelli et al. 1989; Slate } \\
\text { et al. 2007; Padilla } 2020\end{array}$ & $\begin{array}{l}60 / 85 / \\
59 / 44\end{array}$ & $\begin{array}{l}1-100 * \\
10 \text {-point } * \\
6 \text {-point }\end{array}$ & $0.70-0.97$ \\
\hline 2 & $\begin{array}{l}\text { Hassles Scale/Police } \\
\text { Daily Hassles Scale }\end{array}$ & HS/PDHS & $\begin{array}{l}\text { Kanner et al. 1981; Hart et al. } \\
1993\end{array}$ & $117 / 86$ & 5-point & 0.91 \\
\hline 3 & Police Daily Uplift scale & PDUS & $\begin{array}{l}\text { Kanner et al. 1981; Hart et al. } \\
1993\end{array}$ & 82 & 5-point & 0.77 \\
\hline 4 & $\begin{array}{l}\text { Sources of stress among } \\
\text { supervisory police } \\
\text { officers }^{\mathrm{a}}\end{array}$ & & Cooper et al. 1982 & 98 & 6-point & \\
\hline 5 & $\begin{array}{l}\text { Items Composing } \\
\text { Stressor, Support, and } \\
\text { Stress Scales }\end{array}$ & & $\begin{array}{l}\text { Cullen et al. } 1982 \text {, cited in } \\
\text { Cullen et al. } 1985\end{array}$ & 38 & 7-point & $0.64-0.75$ \\
\hline 6 & $\begin{array}{l}\text { Law Enforcement Critical } \\
\text { Life Events Scale }\end{array}$ & $\begin{array}{l}\text { LECLES, } \\
\text { LECLES-R }\end{array}$ & $\begin{array}{l}\text { Sewell 1983; (Sewell 1980); } \\
\text { Coman et al. 1991a, b }\end{array}$ & $137 / 128$ & $6 *$ & $\geq 0.70$ \\
\hline 7 & $\begin{array}{l}\text { Situation Stress } \\
\text { Inventory }^{\mathrm{b}}\end{array}$ & SSI & $\begin{array}{l}\text { Gudjonsson and Adlam } \\
\text { 1983, cited in Gudjonsson } \\
\text { and Adlam } 1985\end{array}$ & 45 & $1-100$ & \\
\hline 8 & $\begin{array}{l}\text { Peace Officer Standards } \\
\text { and Training }\end{array}$ & POST & $\begin{array}{l}\text { Georgia's Peace Officer } \\
\text { Standards and Training } \\
\text { Council 1983, cited in } \\
\text { Anson et al. } 1997\end{array}$ & 37 & 5-point & \\
\hline 9 & Police Stress Inventory & PSI & $\begin{array}{l}\text { Lawrence 1984; Lord et al. } \\
1991\end{array}$ & $60 / 40$ & $\begin{array}{l}\text { 3-point* } \\
\text { 5-point* }\end{array}$ & 0.86 \\
\hline 10 & Job Stress Rating Scale & JSRS & $\begin{array}{l}\text { Taiwo 1985, cited in Oyefese } \\
1989\end{array}$ & 11 & 6-point & 0.85 \\
\hline 11 & $\begin{array}{l}\text { Occupational Stress } \\
\text { Inventory }\end{array}$ & OSI & $\begin{array}{l}\text { Joseph and Dharmangadan } \\
\text { 1988, cited in Dharmangadan } \\
1988\end{array}$ & 120 & 5-point & $0.67-97$ \\
\hline 12 & $\begin{array}{l}\text { Police stressors and felt } \\
\text { stress inventory }\end{array}$ & & Brown and Campbell 1990 & 54 & 4-point* & \\
\hline 13 & $\begin{array}{l}\text { Identify principal sources } \\
\text { of stress }^{\mathrm{a}}\end{array}$ & & Crank and Caldero 1991 & 1 & Open & \\
\hline 14 & $\begin{array}{l}\text { Stress questionnaire } \\
\text { developed by reviews }\end{array}$ & & Bartol et al. 1992 & 87 & 5-point & \\
\hline 15 & Police Stress Inventory & & $\begin{array}{l}\text { Suresh 1992, cited in Nandini } \\
\text { et al. } 2015\end{array}$ & 80 & & 0.75 \\
\hline 16 & $\begin{array}{l}\text { Stress factors and effects } \\
\text { of police work }{ }^{\mathrm{a}}\end{array}$ & & Savery et al. 1993 & 30 & 7-point & \\
\hline 17 & Police Stress Scale & & $\begin{array}{l}\text { Beehr et al. 1995, cited in } \\
\text { Gershon et al. } 2009\end{array}$ & 25 & 5-point* & \\
\hline 18 & $\begin{array}{l}\text { Indices of Institutional } \\
\text { and Organizational } \\
\text { Variables }\end{array}$ & & Crank et al. 1995 & 18 & 5-point & $0.69-0.93$ \\
\hline 19 & Officer Stress Survey & OSS & Lord 1996 & 86 & 5-point & $0.16-0.92$ \\
\hline 20 & Sources of acute stress ${ }^{\mathrm{ab}}$ & & Anshel et al. 1997 & 17 & 5-point* & \\
\hline 21 & $\begin{array}{l}\text { Police Stress } \\
\text { Questionnaire }\end{array}$ & PSQ & Biggam et al. 1997 & 36 & 5-point & \\
\hline 22 & $\begin{array}{l}\text { Police Officer } \\
\text { Questionnaire }\end{array}$ & POQ & $\begin{array}{l}\text { Delprino et al. } 1997 \text { cited in } \\
\text { Rose and Unnithan } 2015\end{array}$ & 3 & 5-point & 0.814 \\
\hline 23 & $\begin{array}{l}\text { Boston Police Officer } \\
\text { Survey }\end{array}$ & BPOS & $\begin{array}{l}\text { Police Dep. Research Office, } \\
\text { Boston 1997, cited in Garcia } \\
\text { et al. } 2004\end{array}$ & 21 & 100 & 0.89 \\
\hline 24 & $\begin{array}{l}\text { Indications of } \\
\text { experienced events } * b\end{array}$ & & Robinson et al. 1997 & 25 & 3-point & \\
\hline
\end{tabular}


Table 2 (continued)

\begin{tabular}{|c|c|c|c|c|c|c|}
\hline No & Name & Abbreviation & Author & Number of items & Response scale $(*)$ & Cronbach's alpha (a) \\
\hline 25 & Policing Event Scale ${ }^{\mathrm{b}}$ & PES & Brown et al. 1999 & 28 & 4-point* & \\
\hline 26 & $\begin{array}{l}\text { Stressful and rewarding } \\
\text { work aspects }{ }^{\mathrm{a}}\end{array}$ & & Kop et al. 1999 & $20+13$ & open & \\
\hline 27 & $\begin{array}{l}\text { Police Perceived Stress } \\
\text { Survey }\end{array}$ & & $\begin{array}{l}\text { Laufersweiler-Dwyer and } \\
\text { Dwyer } 2000\end{array}$ & $50+14$ & 4 point $*$ & $0.84-0.86$ \\
\hline 28 & $\begin{array}{l}\text { Jong Mok Lee Scale of } \\
\text { Police Stress }\end{array}$ & & $\begin{array}{l}\text { Jong Mok Lee 2002, cited in } \\
\text { Lee } 2002\end{array}$ & 31 & 5-point & 0.761 \\
\hline 29 & $\begin{array}{l}\text { Work Environment } \\
\text { Inventory }\end{array}$ & WEI & $\begin{array}{l}\text { Liberman et al. 2002; Marmar } \\
\text { et al. } 2006\end{array}$ & 68 & 5-point & 0.92 \\
\hline 30 & $\begin{array}{l}\text { Law Enforcement Work } \\
\text { Assessment Survey }\end{array}$ & LEWA & $\begin{array}{l}\text { Scott 2002, cited in Scott } \\
2004\end{array}$ & 24 & 10-point & $0.76-0.84$ \\
\hline 31 & $\begin{array}{l}\text { Officers' perception of } \\
\text { stress* }\end{array}$ & & $\begin{array}{l}\text { Taylor Greene and del Carmen } \\
2002\end{array}$ & 5 & 5-point & 0.87 \\
\hline 32 & Police Stress Inventory & PSI & $\begin{array}{r}\text { Pienaar and Rothmann } 2003 \\
\text { cited in Pienaar et al. } 2007\end{array}$ & 44 & 9-point & $0.89-0.92$ \\
\hline 33 & $\begin{array}{l}\text { Law Enforcement Officer } \\
\text { Stress Survey }\end{array}$ & $\begin{array}{l}\text { LEOSS } \\
\text { LEOSS-R }\end{array}$ & $\begin{array}{l}\text { Van Hasselt et al. 2003; Van } \\
\text { Hasselt et al. 2008; Can } \\
\text { et al. } 2015\end{array}$ & $25 / 18$ & 5-point* & $\begin{array}{l}0.923 \\
0.74-0.87\end{array}$ \\
\hline 34 & $\begin{array}{l}\text { Police Stress } \\
\text { Questionnaire }\end{array}$ & PSQ & $\begin{array}{l}\text { Ranta 2004, cited in Ranta } \\
\text { and Sud } 2008\end{array}$ & 45 & & \\
\hline 35 & $\begin{array}{l}\text { Norwegian Police Stress } \\
\text { Survey }\end{array}$ & NPSS & Berg et al. 2005 & 10 & $1-100 *$ & $0.76-0.83$ \\
\hline 36 & $\begin{array}{l}\text { Work stressors among } \\
\text { Israeli police }^{\mathrm{a}}\end{array}$ & & $\begin{array}{l}\text { Malach-Pines and Keinan } \\
2005\end{array}$ & 30 & 5-point & 0.94 \\
\hline 37 & $\begin{array}{l}\text { Police Stress } \\
\text { Questionnaire-Op }\end{array}$ & PSQ-Op & $\begin{array}{l}\text { McCreary and Thompson } \\
\text { 2006; McCreary et al. } 2017\end{array}$ & 20 & 7-point & $0.90-0.94$ \\
\hline 38 & $\begin{array}{l}\text { Police Stress } \\
\text { Questionnaire-Org }\end{array}$ & PSQ-Org & $\begin{array}{l}\text { McCreary and Thompson } \\
\text { 2006; McCreary et al. } 2017\end{array}$ & 20 & 7-point & $0.89-0.93$ \\
\hline 39 & $\begin{array}{l}\text { Female officer stress } \\
\text { sources }^{\mathrm{a}}\end{array}$ & & Thompson et al. 2006 & 16 & 5-point & \\
\hline 40 & $\begin{array}{l}\text { Antoniou Police Stress } \\
\text { Inventory }\end{array}$ & APSI & Karanika-Murray et al. 2009 & 55 & 6-point & 0.86 \\
\hline 41 & $\begin{array}{l}\text { Public Police - Security } \\
\text { Professionals Stress } \\
\text { Questionnaire }\end{array}$ & QSPS-P & $\begin{array}{l}\text { Gomes 2010, cited in Gomes } \\
\text { et al. } 2016\end{array}$ & 25 & 5-point & $0.86-0.93$ \\
\hline 42 & $\begin{array}{l}\text { Critical Incident History } \\
\text { Questionnaire }\end{array}$ & CIHQ & Weiss et al. 2010 & 34 & 5-point & 0.94 \\
\hline 43 & $\begin{array}{l}\text { Exposure to harassment } \\
\text { and role-specific job } \\
\text { demands and resources }^{\mathrm{a}}\end{array}$ & & Tuckey et al. 2012 & & 5-point & $\geq 0.73$ \\
\hline 44 & $\begin{array}{l}\text { Daily Perceived Stress } \\
\text { Level }^{\mathrm{a}}\end{array}$ & & Korre et al. 2014 & 22 & 10-point* & \\
\hline 45 & $\begin{array}{l}\text { Police Stress } \\
\text { Questionnaire }\end{array}$ & PSQ & Wang et al., 2014 & 22 & 5-point & \\
\hline 46 & $\begin{array}{l}\text { Police Work Experience } \\
\text { Survey }\end{array}$ & PWES & Bishopp et al. 2018 & 24 & $\begin{array}{l}\text { 5-point } \\
\text { 7-point }\end{array}$ & \\
\hline
\end{tabular}

*Response scales measuring both perceived severity and frequency of occurrence

${ }^{a}$ Name given by LR

${ }^{\mathrm{b}}$ Events or situations 
Table 3 Perceived stress measurements

\begin{tabular}{|c|c|c|c|c|c|c|}
\hline No & Perceived stress & Abbreviation & Author & Number of items & Response scale & Cronbach's alpha $(a)$ \\
\hline 1 & $\begin{array}{l}\text { The Social Readjustment } \\
\text { Rating Scale }\end{array}$ & SRRE & $\begin{array}{l}\text { Holmes and Rahe 1967, cited } \\
\text { in Collins and Gibbs } 2003\end{array}$ & 56 & Mixed & $0.89-0.96$ \\
\hline 2 & $\begin{array}{l}\text { Schedule of Recent } \\
\text { Experiences }\end{array}$ & SRE & $\begin{array}{l}\text { Holmes 1967, cited in Burke } \\
\text { et al. } 1984\end{array}$ & 42 & Mixed & $0.70-0.88$ \\
\hline 3 & The Stress Profile & & $\begin{array}{l}\text { Girdano and Everly } 1973, \\
\text { cited in Leitner et al. } 1983\end{array}$ & 9 & & \\
\hline 4 & $\begin{array}{l}\text { Occupational Work } \\
\text { Questionnaire }\end{array}$ & OWQ & Caplan et al. 1975 & 4 & 7-point & 0.78 \\
\hline 5 & Chronic and Episodic Stress & & $\begin{array}{l}\text { Adam 1978; Adams et al. } \\
\text { 1978, cited in Anson and } \\
\text { Bloom } 1988\end{array}$ & 45 & 5 point & \\
\hline 6 & Stress in General Scale & & $\begin{array}{l}\text { Ironson and Smith 1978; } \\
\text { Ironson et al. 1989, cited in } \\
\text { Kohan and O'Connor } 2002\end{array}$ & 5 & 7-point & 0.81 \\
\hline 7 & $\begin{array}{l}\text { Personalized Assessment of } \\
\text { Stress Scale }\end{array}$ & PASS & $\begin{array}{l}\text { Morse and Frost 1979, cited in } \\
\text { Anson et al. } 1997\end{array}$ & 27 & 5-point & \\
\hline 8 & $\begin{array}{l}\text { Frequency of stress symptoms } \\
\text { and feeling after } \text { work }^{\mathrm{a}}\end{array}$ & & Lester and Gallagher 1980 & 3 & 4-point & \\
\hline 9 & Perceived Stress Scale & PSS-14/PSS-10 & $\begin{array}{l}\text { Cohen et al. 1983; Cohen and } \\
\text { Williamson 1988, cited in } \\
\text { Graf } 1986\end{array}$ & $14 / 10$ & 5-point & 0.85 \\
\hline 10 & $\begin{array}{l}\text { Presumptive Stressful Life } \\
\text { Event Scale }\end{array}$ & PSLE & $\begin{array}{l}\text { Singh et al. 1984, cited in Roz } \\
\text { and Raval } 2017\end{array}$ & 51 & & 0.80 \\
\hline 11 & Visual Analog Scale & VAS & $\begin{array}{l}\text { Arnetz et al. } 1985 \text {, cited in } \\
\text { Arnetz et al. } 2009\end{array}$ & 1 & $1-100$ & \\
\hline 12 & Subjective stress ${ }^{\mathrm{a}}$ & & $\begin{array}{l}\text { Motowidlo et al., 1986, cited } \\
\text { in Young } 1994\end{array}$ & 4 & 5-point & $0.79-0.896$ \\
\hline 13 & NASA Task Load Index & NASA TLX & $\begin{array}{l}\text { Hart and Staveland 1988, cited } \\
\text { in Chiorri et al. } 2015\end{array}$ & 6 & 20-point & Low \\
\hline 14 & Stress/Energy Questionnaire & & $\begin{array}{l}\text { Kjellberg and Iwanowski } \\
\text { 1989, cited in Garcia et al. } \\
2017\end{array}$ & 12 & 6-point & $\begin{array}{l}\text { Stress: } 0.72 \\
\text { Energy: } 0.53\end{array}$ \\
\hline 15 & Stress Appraisal Measure & SAM & $\begin{array}{l}\text { Peacock and Wong, } 1990 \text {, } \\
\text { Anshel et al., } 1997\end{array}$ & $28 / 33$ & 5-point & $0.71-0.86$ \\
\hline 16 & Work Stress Scale ${ }^{\mathrm{a}}$ & & $\begin{array}{l}\text { Revicki et al. 1993; Gershon } \\
\text { et al. 1995; 1999, cited in } \\
\text { Gershon et al. } 2009\end{array}$ & 11 & 4-point & $0.85-0.91$ \\
\hline 17 & $\begin{array}{l}\text { Measure of Occupational } \\
\text { Stress }^{\mathrm{a}}\end{array}$ & & $\begin{array}{l}\text { Morash and Haarr 1995; Haarr } \\
\text { and Morash, 1999; Morash } \\
\text { et al., } 2006\end{array}$ & 4 & 5-point & 0.77 \\
\hline 18 & $\begin{array}{l}\text { Screening Scale of Chronic } \\
\text { Stress }\end{array}$ & SSCS & $\begin{array}{l}\text { Schulz and Schlotz 1999, } \\
\text { Schulz et al. 2003, 2004, } \\
\text { cited in Strahler and Ziegert } \\
2015\end{array}$ & 12 & 5-point & 0.91 \\
\hline 19 & $\begin{array}{l}\text { Strain-based work-family } \\
\text { conflict }\end{array}$ & & $\begin{array}{l}\text { Carlson et al. 2000, cited in } \\
\text { Qureshi et al. } 2016\end{array}$ & 6 & 5-point & 0.75 \\
\hline 20 & Job stress measurement ${ }^{\mathrm{a}}$ & & $\begin{array}{l}\text { Lait and Wallace 2002, cited } \\
\text { in Tyagi and Dhar } 2014\end{array}$ & 6 & 7-point & 0.88 \\
\hline 21 & Officers' perception of stress ${ }^{\mathrm{a}}$ & & $\begin{array}{l}\text { Taylor Greene and del Carmen } \\
\text { 2002; del Carmen et al. } 2007\end{array}$ & 5 & 5-point & 0.87 \\
\hline 22 & $\begin{array}{l}\text { Management Standard } \\
\text { Indicator Tool — How } \\
\text { stressful do you find your job }\end{array}$ & HSE MS & $\begin{array}{l}\text { HSE Health and Safety Execu- } \\
\text { tive 2004, Edwards 2008, } \\
\text { cited in Allisey et al. } 2014\end{array}$ & 1 & 5-point & \\
\hline 23 & General level of stress ${ }^{\mathrm{a}}$ & & $\begin{array}{l}\text { Malach-Pines and Keinan } \\
2005\end{array}$ & 1 & & \\
\hline
\end{tabular}

${ }^{\mathrm{a}}$ Name given by LR 
Table 4 Measurements of psychological distress, physiological complaints, and/or mood and affect changes

\begin{tabular}{|c|c|c|c|c|c|c|}
\hline No & Name & Abbreviation & Author & Number of items & Response scale & Cronbach's alpha (a) \\
\hline 1 & Mental Health Index & MHI & Langner 1962 & 22 & 2-point & 0.77 \\
\hline 2 & $\begin{array}{l}\text { Subjective Units of Distress } \\
\text { Scale }\end{array}$ & SUDS & $\begin{array}{l}\text { Wolpe } 1969 \text { cited in Taverniers } \\
\text { et al. } 2011\end{array}$ & 1 & 10-point & \\
\hline 3 & State Trait Anxiety Inventory & STAI & $\begin{array}{l}\text { Spielberger et al. 1970; 1983, } \\
\text { cited in Coman et al. 1991a, b }\end{array}$ & $20+20$ & 4-point & $0.74-0.86$ \\
\hline 4 & Profile of Mood States & POMS & $\begin{array}{l}\text { McNair et al. 1971, cited in } \\
\text { Garbarino et al. } 2012\end{array}$ & 58 & 5-point & $0.74-0.93$ \\
\hline 5 & General Health Questionnaire & $\begin{array}{l}\text { GHQ12 } \\
\text { GHQ28 }\end{array}$ & $\begin{array}{l}\text { Goldberg and Williams 1988, } \\
\text { cited in Brown et al. } 1999\end{array}$ & $12 / 28$ & 4-point & $0.679-0.91$ \\
\hline 6 & Symptom Checklist & $\begin{array}{l}\text { SCL-90 } \\
\text { SCL-90R }\end{array}$ & $\begin{array}{l}\text { Derogatis 1975; Derogatis and } \\
\text { Lazarus 1994, cited in Van } \\
\text { Patten and Burke } 2001\end{array}$ & $90 / 56$ & 5-point & $0.84-0.97$ \\
\hline 7 & Eight State Questionnaire & $8 \mathrm{SQ}$ & $\begin{array}{l}\text { Curran and Cattell 1976, cited } \\
\text { in Nathawat and Dadarwal } \\
2014\end{array}$ & 96 & 4-point & \\
\hline 8 & $\begin{array}{l}\text { Short Adjective Checklist } \\
\text { Stress Arousal Checklist S } \\
\text { Stress Arousal Checklist A }\end{array}$ & $\begin{array}{l}\text { SACL } \\
\text { SACL-S } \\
\text { SACL-A }\end{array}$ & $\begin{array}{l}\text { Mackay et al. 1978; Gotts and } \\
\text { Cox 1988, cited in Hart et al. } \\
1993\end{array}$ & $4 / 18 / 12$ & $\begin{array}{l}\text { 4-point } \\
\text { 4-point } \\
\text { 4-point }\end{array}$ & $\begin{array}{l}0.92 \\
0.93 \\
0.88\end{array}$ \\
\hline 9 & $\begin{array}{l}\text { Psycho-Physiology Anxiety } \\
\text { Reactions }\end{array}$ & PAR & $\begin{array}{l}\text { Rose et al. 1978, cited in Lord } \\
\text { et al. } 1991\end{array}$ & 19 & & \\
\hline 10 & Impact of Event Scale & IES/IES-R & $\begin{array}{l}\text { Horowitz et al. } 1979 \text {, cited in } \\
\text { Weiss et al. } 1995\end{array}$ & $15 / 22$ & 5-point & $0.87-0.952$ \\
\hline 11 & Willcher test & & $\begin{array}{l}\text { Willcher 1980, cited in Lester } \\
\text { and Solis } 1981\end{array}$ & 20 & 4-point & \\
\hline 12 & Brief Symptom Inventory & BSI & $\begin{array}{l}\text { Derogatis and Melisaratos 1983, } \\
\text { cited in Mearns and Mauch } \\
1998\end{array}$ & 53 & 5-point & $0.49-0.97$ \\
\hline 13 & $\begin{array}{l}\text { Positive And Negative Affect } \\
\text { Schedule }\end{array}$ & PANAS & $\begin{array}{l}\text { Diener and Emmonds 1984, } \\
\text { Watson et al. } 1988 \text { cited in } \\
\text { Hart et al. } 1994\end{array}$ & $10+10$ & 5-point & $\begin{array}{l}\text { PA: } 0.86-0.90 \\
\text { NA: } 0.84-87\end{array}$ \\
\hline 14 & Work Stress Scale ${ }^{\mathrm{x}}$ & & Cullen et al. 1985 & 6 & 7-point & 0.78 \\
\hline 15 & $\begin{array}{l}\text { Emotional Stress Reaction } \\
\text { Questionnaire }\end{array}$ & ESRQ & $\begin{array}{l}\text { Larsson 1987, cited in Larsson } \\
\text { et al. } 1988\end{array}$ & 18 & 4-point & $0.66-81$ \\
\hline 16 & $\begin{array}{l}\text { Psychosomatic Complaints } \\
\text { Scale of Stress }\end{array}$ & PSCSS & $\begin{array}{l}\text { Greller and Parsons 1988, cited } \\
\text { in Greller and Parsons } 1992\end{array}$ & 31 & 5-point & 0.91 \\
\hline 17 & $\begin{array}{l}\text { Kessler Psychological Distress } \\
\text { Scale }\end{array}$ & K10/KPDS & $\begin{array}{r}\text { Kessler and Mroczek 1994, } \\
\text { cited in Noblet et al. } 2009\end{array}$ & 10 & 5-point & $0.93-0.94$ \\
\hline 18 & $\begin{array}{l}\text { Negative Attitude to Emotional } \\
\text { Expression Scale }\end{array}$ & & $\begin{array}{c}\text { Joseph et al. 1994, cited in } \\
\text { Brown and Grover } 1998\end{array}$ & 20 & 5-point & 0.91 \\
\hline 19 & $\begin{array}{l}\text { Medical Outcomes Study Short- } \\
\text { Form Health Survey }\end{array}$ & MOS-SF 36 & $\begin{array}{l}\text { Ware et al. 1994, cited in Iwasaki } \\
\text { et al. } 2002\end{array}$ & 36 & 10-point & \\
\hline 20 & $\begin{array}{l}\text { Depression, Anxiety, and Stress } \\
\text { Scale }\end{array}$ & DASS & $\begin{array}{l}\text { Lovibond and Lovibond, 1995, } \\
\text { cited in Richmond et al. } 1998\end{array}$ & $42 / 21$ & 4-point & $0.74-0.94$ \\
\hline 21 & Distress Thermometer & DT & $\begin{array}{l}\text { Roth et al. 1998, cited in } \\
\text { Acquadro Maran et al. } 2014\end{array}$ & 36 & 10-point & \\
\hline 22 & Rapid Stress Assessment Scale & RSA & $\begin{array}{l}\text { Tarsitani and Biondi 1999, cited } \\
\text { in Pancheri et al. } 2002\end{array}$ & 15 & 4-point & $0.70-0.92$ \\
\hline 23 & Patient Health Questionnaire & PHQ & $\begin{array}{l}\text { Kroenke et al. } 2002 \text {, cited in } \\
\text { Hall et al. } 2010\end{array}$ & $9 / 15$ & 3-point & \\
\hline 24 & $\begin{array}{l}\text { Lipp Stress Symptoms } \\
\text { Inventory }\end{array}$ & (L)SSI & Lipp 2000, cited in Lipp 2009 & 53 & 2-point & 0.42 \\
\hline 25 & Toulouse Stress Scale & TSS & $\begin{array}{l}\text { Bailly et al. 2004, cited in } \\
\text { Bonnet-Suard et al. } 2005\end{array}$ & 30 & 5-point & 0.83 \\
\hline 26 & $\begin{array}{l}\text { Secondary Traumatic Stress } \\
\text { Scale }\end{array}$ & STS & $\begin{array}{l}\text { Bride et al. 2004, cited in Craun } \\
\text { et al. } 2014\end{array}$ & 17 & 5-point & 0.91 \\
\hline
\end{tabular}


Table 4 (continued)

\begin{tabular}{|c|c|c|c|c|c|c|}
\hline No & Name & Abbreviation & Author & Number of items & Response scale & Cronbach's alpha ( $a$ ) \\
\hline 27 & Professional Quality of Life & ProQOL & $\begin{array}{l}\text { Stamm 2005; 2010, cited in } \\
\text { Burnett et al. } 2019\end{array}$ & 30 & 5-point & \\
\hline 28 & $\begin{array}{l}\text { Stress of Conscience } \\
\text { Questionnaire }\end{array}$ & SCQ & $\begin{array}{l}\text { Glasberg et al. 2006, cited in } \\
\text { Backteman-Erlanson et al. } \\
2013\end{array}$ & 9 & 7-point & 0.83 \\
\hline 29 & $\begin{array}{l}\text { Psychological Injury Risk } \\
\text { Indicator }\end{array}$ & PIRI & $\begin{array}{l}\text { Winwood et al. 2009; Winwood } \\
\text { et al. } 2012\end{array}$ & 30 & 7-point & 0.83 \\
\hline 30 & Acute Stress Response Scale & ASRS & Yang et al. 2011 & 112 & 2-point & 0.952 \\
\hline
\end{tabular}

and/or revision. The psychometric properties (Cronbach's alpha) are listed, if indicated, in the last column. To enhance the informative value of the reported alpha levels, the table also includes the number of items and length of the response scale. Alpha scores are reported as a fixed number or range from the lowest to the highest subscale, depending on the information available. Full reference details for the content of all measurements in Tables 2, 3, 4 and 5 are included as active electronic links.

Table 2 includes 46 unique police-specific measures, all of which include a greater or lesser number of the two primary stress sources affecting police employees: operational and organisational stressors. About half of the measurements $(n=22)$ indicate satisfactory reliability $(>0.80)$ among the police population. Measurements based on lists of events and situations such as 'Sources of acute stress' (Anshel et al. 1997) or 'Policing Event scale' (PES) (Brown et al. 1999) do not provide reliability estimates. Such scales are considered formative, which implies that high correlations among items are not expected. Experiencing one event does not increase the probability of experiencing another. In all the measurements, respondents were asked to rate the severity and/or frequency of the included stressors or situations.

Police-specific measures are characterised by a desire to capture the stress of a demanding occupation in which individuals face increased challenges and changing roles in their work. The measurements differ in terms of how they are constructed. Some measurements (e.g., 'Police Stress Questionnaire' (PSQ) (Biggam et al. 1997), 'Police Daily Hassles Scale'/ 'Police Daily Uplift Scale' (PDHS/PDUS) (Hart et al. 1993; Kanner et al. 1981), and 'Police Stress Questionnaire-Operative'/ 'Police Stress QuestionnaireOrganisational' (PSQ-Op/PSQ-Org) (McCreary et al. 2017; McCreary and Thompson 2006) organised stressors into two scales in which operational and organisational stressors are measured as latent variables. Other measurements, such as the 'Occupational Stress Inventory' (OSI) (Dharmangadan 1988), 'Police Stress Survey' (PSS) (Martelli et al. 1989; Padilla 2020; Patterson 1992; Slate et al. 2007; Spielberger et al. 1981; White et al. 1985), and 'Police Stress Questionnaire' (PSQ) (Wang et al. 2014), include subscales inside the two segments in a manner similar to multidimensional assessment batteries (see Table 5). In the operational segment, we find questions of physical and psychological danger, dealing with domestic violence, and equipment issues.

In the organisational segment, we find questions concerning lack of support, workload, boredom, personal relations, and external sociopolitical variables (such as public relations, home-work balance, media pressure, and court issues). In addition, some measurements are constructed to investigate stressors in the daily routine, e.g., 'Police Daily Hassles Scale'/ 'Police Daily Uplift Scale PDHS/PDUS' (Hart et al. 1993; Kanner et al. 1981) 'Work Environment Inventory' (WEI) (Liberman et al. 2002; Marmar et al. 2006), and 'Daily Perceived Stress Level' (Korre et al. 2014). Others, such as 'Sources of acute stress' (Anshel et al. 1997) and 'Policing Event scale' (Brown et al. 1999), are concerned with acute stressors. A few measurements include questions regarding responses and outcomes (e.g., burnout, anxiety) or mood changes such as anger, e.g., 'Stress factors and effects of police work' (Savery et al. 1993), 'Police Work Experience Survey' (PWES) (Bishopp et al. 2018), and 'Police Officer Questionnaire' (POQ) (Delprino et al. 1997; Rose and Unnithan 2015).

Overall, police-specific stress measurements have been developed for a variety of purposes ranging from development programs, e.g., the 'Policing Event Scale' (PES) (Brown et al. 1999), 'Police Stress Inventory' (PSI) (Pienaar and Rothmann 2003; Pienaar et al. 2007) and PSS (Martelli et al. 1989; Padilla 2020; Patterson 1992; Slate et al. 2007; Spielberger et al. 1981; White et al. 1985), predicting employees at risk, e.g., 'Police Perceived Stress Survey' (Laufersweiler-Dwyer and Dwyer 2000) or 'Law Enforcement Officer Stress Survey' (LEOSS) (Can et al. 2015; Van Hasselt et al. 2008, 2003), estimating average work distribution, e.g., 'Daily Perceived Stress Level' (Korre et al. 2014), to improving knowledge concerning how to cope, e.g., 'Sources of acute stress' (Anshel et al. 1997).

Table 3 includes 23 measurements assessing perceived stress. Among this group, $48 \%(n=11)$ reported an alpha score over 0.80 . Measurements in this category contain questions in which respondents are explicitly asked about perceived or experienced stress in general, at work, or in specific settings. 
Table 5 Assessment batteries measuring occupational workload/stress/demands

\begin{tabular}{|c|c|c|c|c|c|c|}
\hline No & Name of measurement & Abbreviation & Author & Number of items & Response scale & Cronbach's alpha $(a)$ \\
\hline 1 & Job demand stress & & $\begin{array}{l}\text { House and Rizzo 1972, cited in } \\
\text { White and Marino } 1983\end{array}$ & 26 & 2-point & \\
\hline 2 & Quantitative Workload Scale & QWS & $\begin{array}{l}\text { Caplan et al. } 1975 \text {, cited in } \\
\text { Pendergrass and Ostrove } 1984\end{array}$ & 117 & 5-point & $0.575-0.86$ \\
\hline 3 & $\begin{array}{l}\text { Personalized Assessment } \\
\text { Stress Scale }\end{array}$ & PASS & $\begin{array}{l}\text { Morse and Frost } 1979 \text { cited in } \\
\text { Anson et al. } 1997\end{array}$ & 27 & 5-point & \\
\hline 4 & $\begin{array}{l}\text { Cherniss model of work } \\
\text { stress }\end{array}$ & & $\begin{array}{l}\text { Cherniss 1980, cited in Burke } \\
\text { et al. } 1984\end{array}$ & 14 & & $0.86-0.88$ \\
\hline 5 & $\begin{array}{l}\text { Occupational Stress Index } \\
\text { Occupational Stress } \\
\text { Inventory }\end{array}$ & $\begin{array}{l}\text { OSI } \\
\text { OSI-R }\end{array}$ & $\begin{array}{l}\text { Srivastava and Singh 1981, 1984, } \\
\text { cited in Roz and Raval 2017; } \\
\text { Osipow and Spokane 1984, 1998, } \\
\text { cited in Yu et al. 2016; Joseph } \\
\text { and Dharmangadan 1988; cited } \\
\text { in Dharmangadan } 1988\end{array}$ & $46 / 57 / 120 / 140$ & $\begin{array}{l}\text { 5-point } \\
\text { 5-point }\end{array}$ & $\begin{array}{l}0.9-0.935 \\
0.67-0.97\end{array}$ \\
\hline 6 & $\begin{array}{l}\text { Organisational Role Stress } \\
\text { Scale }\end{array}$ & ORS(S) & $\begin{array}{l}\text { Pareek 1983, cited in Tundwal } \\
\text { and Behmani } 2016\end{array}$ & 50 & 5-point & \\
\hline 7 & $\begin{array}{l}\text { ERI-Effort Reward } \\
\text { Imbalance }\end{array}$ & ERI & $\begin{array}{l}\text { Siegrist } 1984,2002 \text {, cited in } \\
\text { Garbarino et al. } 2011\end{array}$ & $35 / 23$ & 4/5-point & $0.82 / 0.89 / 0.79$ \\
\hline 8 & $\begin{array}{l}\text { Masculine Gender Role } \\
\text { Stress }\end{array}$ & MGRS & $\begin{array}{l}\text { Eisler and Skidmore 1987, cited } \\
\text { in Tang and Lau } 1996\end{array}$ & 40 & 6-point & $0.73-0.90$ \\
\hline 9 & $\begin{array}{l}\text { Occupational Stress } \\
\text { Indicator/Index }\end{array}$ & $\begin{array}{l}\text { OSI } \\
\text { OSI-2 }\end{array}$ & $\begin{array}{l}\text { Cooper et al. 1988, cited in } \\
\text { Robertson et al. 1990; Evers } \\
\text { et al. 2000; Siu } 2002\end{array}$ & $167 / 61 / 46 / 94$ & 5/6-point & $0.73-97$ \\
\hline 10 & Work Stress Inventory & WSI & $\begin{array}{r}\text { Barone et al. 1988, cited in } \\
\text { Simons and Barone } 1994\end{array}$ & 40 & 5/4-point & $\geq 0.83$ \\
\hline 11 & $\begin{array}{l}\text { Life Stressors and Social } \\
\text { Resources Inventory }\end{array}$ & LISRES & $\begin{array}{l}\text { Moos et al. 1988, cited in Iwasaki } \\
\text { et al., } 2002\end{array}$ & 85 & 4/5-point & \\
\hline 12 & $\begin{array}{l}\text { Professional Life Stress } \\
\text { Scale }\end{array}$ & PLSS & $\begin{array}{l}\text { Fontana 1989, cited in Ramakrishnan } \\
\text { et al. } 2013\end{array}$ & 24 & 3-point & \\
\hline 13 & Feminine Gender Role Stress & FGRS & $\begin{array}{l}\text { Gillespie and Eisler 1992, cited in } \\
\text { Tang and Lau } 1996\end{array}$ & 39 & 6-point & $0.73-0.90$ \\
\hline 14 & $\begin{array}{l}\text { Occupational Stress } \\
\text { Questionnaire }\end{array}$ & OSQ & $\begin{array}{l}\text { Gmelch and Chan 1994, cited in } \\
\text { Singh and Kar } 2015\end{array}$ & 78 & 5-point & $0.92-0.99$ \\
\hline 15 & Job Stress Survey & JSS & $\begin{array}{l}\text { Spielberger and Reheiser 1994, } \\
\text { cited in Haisch and Meyers } \\
2004\end{array}$ & $60 / 30$ & 9 point & $0.83-0.92$ \\
\hline 16 & $\begin{array}{l}\text { Mental Health Professional } \\
\text { Stress Scale }\end{array}$ & MHPSS & $\begin{array}{l}\text { Cushway et al. 1996, cited in } \\
\text { Zefferino et al. } 2006\end{array}$ & 42 & 4-point & $0.60-0.87$ \\
\hline 17 & $\begin{array}{l}\text { Queensland Public Agency } \\
\text { Staff Survey }\end{array}$ & QPASS & $\begin{array}{l}\text { Hart et al. } 1996 \text { cited in Dollard } \\
\text { et al. } 2013\end{array}$ & 5 & 5-point & \\
\hline 18 & $\begin{array}{l}\text { Job Content Questionnaire } \\
\text { Demand/Control/Support } \\
\text { Questionnaire }\end{array}$ & $\mathrm{JCQ} / \mathrm{DCS}$ & $\begin{array}{l}\text { Karasek et al. 1998; Sanne et al., } \\
\text { 2005, cited in Garbarino et al. } \\
2011\end{array}$ & $31 / 17$ & 4-point & $\geq 0.700 .71 / 0.65 / 0.84$ \\
\hline 19 & $\begin{array}{l}\text { Pressure Management } \\
\text { Indicator }\end{array}$ & PMI & $\begin{array}{l}\text { Williams and Cooper 1998, cited } \\
\text { in Kirkcaldy and Shephard } \\
2001\end{array}$ & 120 & 5-point & $0.64-0.89$ \\
\hline 20 & Dutch VBBA & & $\begin{array}{l}\text { van Veldhoven et al. 1997, } 2002 \\
\text { cited in Van den Broeck et al. } \\
2010\end{array}$ & 9 & 4-point & $\geq 0.91$ \\
\hline 21 & $\begin{array}{l}\text { Perceived Job Stress } \\
\text { Questionnaire }\end{array}$ & PJSQ & $\begin{array}{l}\text { Dudek et al. } 1999 \text { cited in } \\
\text { Oginska-Bulik } 2005\end{array}$ & 55 & & \\
\hline 22 & Baltimore, Maryland & & $\begin{array}{l}\text { Gershon 1999, cited in Gershon } \\
\text { et al. } 2009\end{array}$ & 132 & Mixed & 0.872 \\
\hline 23 & $\begin{array}{l}\text { Trier Inventory for the } \\
\text { Assessment of Chronic } \\
\text { Stress }\end{array}$ & TICS & $\begin{array}{l}\text { Schulz and Schlotz 1999; Schulz } \\
\text { et al. 2003, } 2004 \text { cited in Gerber } \\
\text { et al. } 2013\end{array}$ & 57 & 5-point & \\
\hline
\end{tabular}


Table 5 (continued)

\begin{tabular}{|c|c|c|c|c|c|c|}
\hline No & Name of measurement & Abbreviation & Author & Number of items & Response scale & Cronbach's alpha $(a)$ \\
\hline 24 & $\begin{array}{r}\text { Brief Job Stress } \\
\text { Questionnaire }\end{array}$ & BJSQ & $\begin{array}{l}\text { Shimomitsu et al. } 2000 \text { cited in } \\
\text { Shiozaki et al. } 2017\end{array}$ & 62 & 4-point & \\
\hline 25 & $\begin{array}{l}\text { Management Standards } \\
\text { Indicator Tool }\end{array}$ & $\begin{array}{l}\text { MS Indicator } \\
\text { Tool }\end{array}$ & $\begin{array}{l}\text { Cousins et al. } 2004 \text { cited in } \\
\text { Edwards et al. } 2008\end{array}$ & 35 & 5-point & $0.78-0.88$ \\
\hline 26 & $\begin{array}{l}\text { A Shortened Stress } \\
\text { Evaluation Tool }\end{array}$ & ASSET & $\begin{array}{l}\text { Faragher et al. } 2004 \text { cited in } \\
\text { Johnson et al. } 2005\end{array}$ & $46 / 6$ & 6-point & $0.762-0.87$ \\
\hline 27 & $\begin{array}{l}\text { Demand-Induced Strain } \\
\text { Questionnaire }\end{array}$ & DISQ & $\begin{array}{l}\text { de Jonge et al. } 2004 \text { cited in Bova } \\
\text { et al. } 2013\end{array}$ & 30 & 5-point & \\
\hline 28 & $\begin{array}{l}\text { Job-Demands Resources } \\
\text { Scale }\end{array}$ & JDRS & $\begin{array}{l}\text { Rothmann } 2006 \text { cited in Rothmann } \\
\text { et al. } 2006\end{array}$ & 48 & 4-point & $0.71-0.90$ \\
\hline 29 & $\begin{array}{l}\text { Korean Occupational Stress } \\
\text { Scale }\end{array}$ & KOSS & Chang et al. 2005 & $43 / 24$ & 4-point & \\
\hline 30 & DECORE-21 & DECORE & $\begin{array}{l}\text { Luceño-Moreno and Martín- } \\
\text { Garcia } 2008 \text { cited in Talavera- } \\
\text { Velasco et al. } 2018\end{array}$ & $44 / 21$ & 5-point & $0.81-0.85$ \\
\hline
\end{tabular}

Some measurements are constructed to assess the perception of overall occupational stress. Typical items could be 'I feel a great deal of stress because of my job ', 'When I'm at work I often feel tense or uptight' or 'I feel pressured', for example, in 'Occupational Work Questionnaire' (OWQ) (Caplan et al. 1975), 'Measure of Occupational Stress' (Haarr and Morash 1999; Morash et al. 2006; Morash and Harr 1995), and 'Subjective Stress' (Motowidlo et al. 1986; Young 1994). Other measurements examine overall, global stress, not necessarily stress linked specifically to work, with items such as 'How often have you felt that you were unable to control important things in your life', 'In the last month, how often have you been upset because of something that happened unexpectedly', or 'In the past year, how would you rate the amount of stress in your life', e.g., 'Perceived Stress Scale' (PSS-14, PSS-10) (Cohen et al. 1983; Cohen and Williamson 1988; Graf 1986) and 'Personalized Assessment of Stress Scale' (PASS) (Anson et al. 1997). Table 3 also includes measurements assessing experiences of life events such as the death of a loved one, marital issues, and economic difficulties, e.g., 'Stress Appraisal Measure' (SAM) (Anshel et al. 1997; Peacock and Wong 1990), 'The Social Readjustment Rating Scale' (SRRE) (Collins and Gibbs 2003; Holmes and Rahe 1967) and 'Schedule of Recent Experiences' (SRE) (Burke et al. 1984) and acute stress experiences, e.g., 'Chronic and Episodic Stress' (Anson and Bloom 1988), 'Measure of Occupational Stress' (Haarr and Morash 1999; Morash et al. 2006; Morash \& N. Haarr, 1995), and 'Screening Scale of Chronic Stress' (SSCS) (Strahler and Ziegert 2015).

Table 4 includes 30 measurements. The measurements in this category investigate the presence of stress through impairment of functioning due to common types of psychiatric, physiological, or behavioural symptoms or mood changes resulting from prolonged or acute stress exposure among police employees. Sixty-three percent $(n=19)$ of the measurements reported Cronbach's alpha score over 0.80. Horrors from the world wars contributed to the growing scholarly agreement concerning a strong relationship between exposure to stress and the subsequent onset of various forms of illness. Additionally, studies conducted during the 1960s identified personal and situational factors mediating the extent of illness.

Examples of instruments measuring physiological outcomes such as headaches, back pain, and ulcers include, e.g., 'Psychosomatic Complaints Scale of Stress' (PSCSS) (Greller and Parsons 1988, 1992) 'Lipp Stress Symptoms Inventory' (L)SSI) (Lipp 2009), 'Psycho-Physiology Anxiety Reactions' (PAR) (Lord et al. 1991), and 'Medical Outcomes Study Short-Form Health Survey’ (MOS-SF36) (Iwasaki et al. 2002). Examples of instruments measuring psychological outcomes, such as anxiety, depression, and discomfort, include the 'Symptom Checklist' (SCL-90) (Van Patten and Burke 2001), Brief Symptom Inventory (Derogatis and Melisaratos 1983; Mearns and Mauch 1998), 'General Health Questionnaire' (GHQ) (Brown et al. 1999), 'Depression, Anxiety, and Stress Scale' (DASS) (Lovibond and Lovibond 1995; Richmond et al. 1998), 'State-Trait Anxiety Scale' (STAI) (Coman et al. 1991a; Spielberger et al. 1970), and 'Psychological Injury Risk Indicator' (PIRI) (Winwood et al., 2012, 2009). Certain measures also investigate behavioural symptoms such as arousal, intrusion, avoidance, sleep problems, excessive drinking, and withdrawal, for example, 'Negative Attitudes to Emotional Expression Scale' (Brown and Grover 1998), 'Toulouse Stress Scale' (TSS) (Bonnet-Suard et al. 2005), 'Secondary Traumatic Stress Scale' (STS) (Bride et al. 2004; Craun et al. 2014), 'Impact of Event Scale' (IES) (Horowitz et al. 1979; Weiss et al. 1995), and 'Willcher test' (Lester and Solis 1981). In addition, mood and affect changes 
such as aggression, anger, and sadness are measured, e.g., 'Emotional Stress Reaction Questionnaire' (ESRQ) (Larsson et al. 1988), 'Work Stress' (Cullen et al. 1985), 'Eight State Questionnaire' (8SQ) (Nathawat and Dadarwal 2014), 'Stress Arousal Checklist-Stress'/'Stress Arousal Checklist-Arousal (SACL-S, SACL-A) (Hart et al. 1993; Mackay et al. 1978), and 'Positive and Negative Affect Schedule' (PANAS) (Hart et al. 1994; Watson et al. 1988). Measurements of acute stress reactions, e.g., 'Rapid Stress Assessment' (RSA) (Pancheri et al. 2002) and 'Acute Stress Response Scale' (ASRS) (Yang et al. 2011), are also included. Measurements in this category examine the presence of a variety of adverse health reactions, symptoms, and mood changes and are not primarily developed to measure exposure to occupational stress.

Table 5 includes 30 batteries of measurements. In this group, $63 \%(n=19)$ reported Cronbach's alpha score, most of which were above 0.80 . Batteries of measurements are mostly comprehensive and multidimensional. These batteries are constructed with several subscales and developed for general use in studies concerning occupational stress. Measurements in this category typically embrace what Cooper and Robertson, cited in Hesketh et al. (2019), refer to as the 'six essentials', which are resources and communications, control, work relationships, balanced workloads, job security, change, and job conditions. Most measurements also include sociopolitical issues such as home-work issues, political pressure, relations with the public or society, occupational status, and organisational changes. Thus, measurements in this category gauge stress in the form of working demands and requirements of organisations in general.

\section{Discussion}

This paper aims to provide an overview of existing and applied stress measures among employees of the police, as well as an overview of the rigour of these measures. According to the scoping review, 129 unique subjective measurements have been used to assess stress among police (see Tables 2, 3, 4 and 5), and these measures appear to work well, with a slight majority of measurements having Cronbach's alpha over 0.80 .

One hundred twenty-nine measurements represent four categories that address different aspects of stress as a phenomenon and the transactional framework of stress. The first category is police-specific, followed by perceived stress, psychological and physiological outcomes (including mood and affect changes), and assessment batteries. Combined, these categories illustrate different assets of the complex interactions among stimuli (i.e., stressors referring to conditions causing subsequent reactions), appraisals of stress (i.e., an individual's perception of and relationship to stressors), and responses in the form of outcomes and mood changes. As a result, these categories highlight different parts of the transactional model. Robinson $(2018$, p. 6) noted that 'the delineation of stress into primary and secondary appraisals has moved stress research away from the purely physiological exploration of stimulus and response in the 1960s and 1970s and more towards cognitive mediation involving numerous factors and feedback loops'. Combinations of measures may improve the study of stress among police by allowing researchers to investigate the ways in which the cognitive process of appraisal acts as a mediator when confronted with a stressor (Robinson 2018). This approach may enhance the current situation, as some researchers have argued that studies of stress among police mainly focus on the nature of stressors without interpreting their results through a theoretical framework of stress (Rodrigues et al. 2019).

Items assessing severity are tightly connected to emotional reactions. This makes it possible to deduce the type of appraisal being produced (i.e., positive-benign, harm-threats, or challenges). Questions of frequency that correspond to the cumulative sum of contextual, situational, or private disturbances further inform the appraisal process. Researchers must examine respondents' personal experiences concerning both the frequency and perceived severity of encounters to interpret the corresponding appraisals (Evans and Coman 1993). According to Anshel et al. (Anshel et al. 1997, p. 349), the primary appraisal dimensions and perceived stressfulness explained more variance than the secondary appraisal scales and the reappraisals. As a result, examining the meaning that individuals attribute to different events contributes to knowledge concerning how secondary appraisals are associated with an individual's overall perceptions of stress. Hence, subjective perspectives, experiences, and perceptions of stress are critical to making long-term contributions to the research concerning work stress.

To summarise, the four categories of measurements complement one another. Police-specific measurements are derived from concrete occupational demands and events and gauge the major operational and organisational occupational requirements of policing. The other three categories focus on general and cross-occupational working demands and outcomes. Because general workplace problems account for a great deal of perceived job stress (Liberman et al. 2002), selected scales from comprehensive and multidimensional assessment batteries often contribute points of overlap and elaborations that are useful in studying working conditions among police. Furthermore, a measurement of perceived stress is often added to capture respondents' experiences of general work-related issues and overall stress in life.

Measurements of psychological and physiological outcomes, including mood and affect changes, are used to verify the presence of stress through various reactions or to 
examine outcomes or mood and affect changes. However, symptoms may have multiple causes, so scales of this kind have shortcomings in regard to interpreting associations and causalities, e.g., according to Gotts and Cox (1988), quoting Mackay et al., mood changes are frequently conflated with stress in the literature (Ermasova et al. 2020).

The majority of measurements in our results are used to assess chronic stress. Measuring acute stress is more difficult and is often conducted through artificial scenarios (Arble et al. 2019; Larsson et al. 1988; Taverniers et al. 2011). When performing simulated stressful tasks, respondents know that no serious consequences will result. Consequently, it is difficult to assure the accuracy of measuring stress originating from the task (Bertilsson et al. 2019; Brisinda et al. 2015). Acute stress is frequently assessed using biomarkers (e.g., saliva, heart rate, blood serum) combined with other measurements (Sandvik et al. 2020). However, both biomarkers and subjective measurements have shortcomings. Regardless of the choice of methods, individual motivations (Ghazinour et al. 2021) and situational cues strongly affect performance, outcomes from biomarkers, and responses (Giessing et al. 2019). It is therefore essential to ensure the ecological validity of the method applied.

\section{Generic or Specific Measurements}

Altogether, measurements from the four categories provide four points of entry to studying stress among police. However, our findings show that most measures are combined when applied to the police. In other words, police-specific measures are applied in conjunction with one or more measures from one of the other main categories. One of the reasons for this combination may be that existing measurements of stress among police include both generic and policespecific measures. Generic assessment batteries, more than police-specific measurements, have evolved in response to generally new knowledge and changes in contextual factors in workplaces (Bliese et al. 2017). Using various types of measurements is a strength, as doing so connects stress among police to the general stress literature, which provides a vital occupational context. Measurements and subscales from different categories possess complementary properties and may successfully be used in conjunction and combination.

Perhaps due to our choice of search terms, most measurements we identified are directed at the negative health effects of stress. With a few notable exceptions (Hart et al. 1993; Kop et al. 1999), most stressors in the measurements we have found appeal to harm-threat appraisals rather than positive-benign and challenging appraisals. Nevertheless, stress has both positive and negative effects. As demonstrated by the upwards slope of the inverted-U curve, positive linear theory views stress as motivating and challenging, whereas the downwards slope follows the negative linear theory, which holds that stress at any level is harmful and reduces performance (Gaines and Van Tubergen 1989). This primary emphasis on detrimental effects may hamper the possibility of gaining more knowledge into job factors that promote and sustain positive forms of stress in policing work (Hart et al. 1994). Larsson et al. (Larsson et al. 1988) found officers to perceive situations as challenging more often than they perceived them to be threatening, suggesting that situations were perceived as controllable or solvable, thereby allowing officers to remain task oriented, confident, and alert. Hence, it would be inappropriate to assume that employees of the police always experience negative stress simply due to exposure. Therefore, conclusions are not straightforward and have several interpretational pitfalls. The identification of positive and negative aspects of work is invaluable for attaining a broader picture that informs both employees and employers of areas on which to focus for improving work conditions and suggesting subsequent interventions (Bakker and Schaufeli 2008; Juniper et al. 2010; Karanika-Murray et al. 2009).

Operational stressors in measurements of policing are mainly the result of police work in the form of physical exposure to citizens, the use of force, and the sense of working with a strong possibility of violence, dangerous events, or death. Notably, most operational stressors result from the aspects of policing that are performed in the streets, i.e., 'real police work' (Reuss-Ianni 2011). This fact remains true even though police work encompasses a wide range of police-specific operational tasks that are demanding in other ways (e.g., preventing and investigating cybercrime, human trafficking, or meeting people undergoing crisis). It may seem that existing police-specific measures support a somewhat outdated narrative concerning police stress. Police-specific measures of stress may profit from a better alignment with the status quo of modern police work in their region of application. It is worth noting that the growth of professional policing worldwide has been accompanied by an increasing number of civilian employees who lack police education (Adams and Mastracci 2020). Regardless of educational background, police employees also collaborate and participate in multiagency efforts, for instance, in online policing (e.g., investigations of sexual abuse and dating fraud).

Studies concerning police stress have repeatedly documented the fact that the most frequent and stressful job factors are related to the overall organisation of work salient to all staff (Shane 2020). Such general working conditions have an impact on the majority of police employees, regardless of the work tasks performed, such as real-life or virtual patrolling, investigative work, contact or dispatch personnel, and counselling or managing (Brown et al. 1996; Galbraith et al. 2021; Kirkcaldy et al. 1998). Police services, like the public sector in general, face heightened demands for efficiency 
and transparency (Andersson and Tengblad 2009). Detrimental ways of organising work are found across occupational settings and are not unique to police work (Bliese et al. 2017; Pagon et al. 2011). Therefore, combining policespecific measurements with general measures of stress may be beneficial when applied to the police. Furthermore, such practice allows for the comparison of results across occupations, which is critical for developing the occupational stress research field (Evans and Coman 1993).

Adapting to the changing societal context of the contemporary world is a key driver in professionalising the police (Cockcroft 2020). This exceedingly complex process causes multiple reforms in police organisations worldwide. The changes have implied a growing need for competence, skills, and expertise (e.g., in social sciences, technology, or languages) that are not necessarily covered by ordinary police training and education (Bjørgo and Damen 2020). Additionally, with the emphasis on the competence needed to perform multiagency policing efforts, it is also anticipated that police services will increase their number of employees with other educational backgrounds, including at the police executive level.

Stressors change as areas of responsibility, roles, and tasks in the police are altered. As Brown et al. (1996) note, rank is an important variable, with staff in supervisory positions showing distinctive patterns of exposure and experience of stress compared to junior officers. Consequently, it is predicted that junior officers report higher levels of operational stress and lower levels of management organisational stress than senior staff. While some police employees perform their work tasks in highly polluted environments, in extreme heat, or in areas with dangerous animals (e.g., traffic police, environmental crime police, border police), other employees increasingly patrol and investigate, whether on or off the internet, highly emotionally demanding environments (e.g., online abuse of children, dating fraud, and domestic violence). The notable differences in the organisation of police work must be accounted for when interpreting the results of stress measurements among police. Models of police stressors should have some validity in terms of their capacity to discriminate meaningfully between groups of employees working in different contexts, with access to different resources, with different educational backgrounds, and in different departments in different parts of the world. With the use of police-specific measurements and assessment batteries in cooperation, police studies may reap benefits from both even though there may be considerable contextual differences.

\section{How the Measurements Work}

Psychometric properties in this scoping review were operationalised by reference to Cronbach's alpha. In this study, the measurements of Cronbach's alpha ranged from 0.16 to 0.97 . A slight majority (54\%) of the measures $(n=70)$ across the four categories had Cronbach's alpha values of approximately 0.80 or over. This fact indicates that, overall, they seem to work rather well. Furthermore, many of the observed differences they produce may be attributed to actual differences among individuals. Thus, 54\% of the 129 measures seem to be consistent in their ability to reproduce similar results across time and space.

However, across the four categories, there were a few measures with low reliability scores $(\leq 0.70)$. A low level of reliability indicates that the observed differences are contaminated by measurement error. Because measurements cannot be valid unless they are reliable, low reliability implies that these measures may not be equally effective at measuring a consistent difference in stress between individuals in the police. However, it should be noted that some of the measures may have been based on a formative scale development strategy (Diamantopoulos and Winklhofer 2001). If this is the case, Cronbach's alpha should not be used as a criterion for scale quality.

As shown in the tables, estimates of Cronbach's alpha scores differ. Reliability is not a fixed property (Keszei et al. 2010) but a function of the measurement, respondents, and circumstances under which a study is conducted. Before a measurement instrument can be used, its measurement properties, i.e., its reliability, validity, and responsiveness, should be assessed and found to be adequate.

Regardless of the assessment method, estimates of reliability should always be scrutinised for inaccuracies, as any chosen method entails biases. For example, known factors affecting the Cronbach's alpha score are its assumptions of uncorrelated errors, nonequal tau equivalence, normality, and unidimensionality (McNeish 2018).

Unidimensionality has to be verified prior to calculating Cronbach's alpha score. The reliability analysis may begin with an inspection of the factor structure. Scales that inadvertently investigate more than one construct are by definition not unidimensional and should not be measured by Cronbach's alpha. Factor loadings and latent variable modelling are increasingly common and may be warranted to measure tau equivalence and whether items vary jointly (DeVellis 2017). Usually, items in scales measure the same construct to different degrees of precision, which becomes visible through factor analysis. Furthermore, the circumstances under which the questionnaire is administered impact reliability and may cause uncorrelated errors where sources other than the construct being measured cause item responses to be related to another. While nonequal tau equivalence and normality of variable distributions cause Cronbach's alpha estimates that are too low, the uncorrelated errors affect estimates of Cronbach's alpha in unpredictable ways. 
Cronbach's alpha is also dependent on scale length. Thus, irrespective of internal consistency, Cronbach's alpha will be high in scales consisting of more than approximately 15 items. In contrast, the shorter the scales, the more Cronbach's alpha reflects the correlations among the items included. Scales with a high Cronbach's alpha might be too interrelated and could indicate redundancy. New prerequisites and new findings or knowledge may change the reliability of measurements. Researchers investigating occupational stress have an imposed obligation to ensure realistic and recognisable wordings and expressions where the chosen stressors reflect the actual working context. Police-specific measurements have evolved in different parts of the world. As they reflect different work settings and conditions, modifications may be necessary before use.

\section{Strengths and Limitations}

This rigorous overview could not have been possible without a meticulous and systematic literature search. Through this search, the content and target of each measure, as well as the ways in which they operate in police settings, have been described.

A limitation of the scoping review, which may have hampered the conclusions and the recommendations drawn, is that studies in languages other than English were excluded. It is also important to note the need for awareness of the context surrounding the measures. Economic, cultural, political, and historical development affect society on both sides of the law, as do climate (Rasdi et al. 2017), geography (Ricciardelli 2018), police education (Bjørgo and Damen 2020), population, crime rate, and the number of police employees (Garcia et al. 2004). Thus, although the measures included in this scoping review are varied, they may be biased, as they predominantly portray the police in a minority of the world (e.g., Northern America and Europe). However, keeping these limitations in mind, this comprehensive review offers a representative view of measures of stress in the police.

Psychometric properties may be measured in several ways other than by Cronbach's alpha. With a broader scope of assessment, we could have included estimates of more measurements. First, single-item measurements do not provide Cronbach's alpha scores, and measurements without Cronbach's alpha scores may have been examined by other methods (e.g., by structural equation modelling, SEM, or McDonald's omega, $\omega)$. On the other hand, reliability differs as the populations and purposes of the study vary. That is, any measurement in the tables may work well and serve its purpose in the context in which it was used, independent of Cronbach's alpha indicated in the tables. Nevertheless, as Cronbach's alpha is a well-known and common way to establish rigour, it served the purpose of this scoping review, in which the aim was to gain a better overview of measurements and their psychometric properties.

\section{Recommendations}

Based on the findings in this scoping review, the following recommendations for conducting studies into stress among police are made.

First, depending on whether the aim of a study is to investigate stress among police across different categories, whether from a generic or a police-specific perspective, measures should be chosen to ensure alignment with theoretical assumptions, the aspect of the stress phenomenon the study aims to investigate, and sufficient psychometric properties.

Second, reliability scores should be evaluated before choosing a measure. In this study, a slight majority of measures had Cronbach's alpha values of approximately 0.80 or over. However, this information was not included in all the records we examined. Measurement should not be chosen based solely on reliability scores (e.g., Cronbach's alpha). We encourage all researchers to conduct reliability tests and interpret the score in light of known biases.

Third, if the aim is to investigate subjective stress experiences from a generic or a police-specific perspective, researchers and practitioners should apply one of the questionnaires that commonly ask respondents to rate the extent to which a situation or stressor is perceived (or not perceived) as stressful. These questionnaires may include police-specific measurements such as Daily Perceived Stress Level (Korre et al. 2014), 'Law Enforcement Critical Life Event Scale' (LECLES) (Coman et al. 1991b; Sewell 1983), 'Law Enforcement Officer Stress Survey' LEOSS (Can et al. 2015; Van Hasselt et al. 2008, 2003), 'Critical Incident History Questionnaire' (CIHQ) (Weiss et al. 2010), or 'Policing Event Scale' PES (Brown et al. 1999). Generic measurements could include 'Subjective Stress' (Motowidlo et al. 1986; Young 1994). Among assessment batteries, 'Work Stress Inventory' (WSI) (Barone et al. 1988), 'Occupational Stress Indicator', OSI (Evers et al. 2000; Robertson et al. 1990; Siu 2002), or the 'Job Stress Survey' (JSS) (Spielberger and Reheiser 1994) could be considered.

Fourth, if the aim is to detect the presence of different police stressors in the context of work stress and demands, police-specific measures such as the 'Police Daily Hassles Scale/Police Daily Uplift Scale', PDHS/ PDUS (Hart et al. 1993; Kanner et al. 1981), and 'Occupational Stress Inventory’, OSI (Dharmangadan 1988) might work well. Among generic measurements, we recommend 'Perceived Stress Scale', PSS-14 or PSS-10 (Cohen et al. 1983; Cohen and Williamson 1988; Graf 1986), 'Job Demands Resource Scale' (JDRS) (Rothmann et al. 2006), 'A Short Stress Evaluation Tool' (ASSET) 
(Faragher et al. 2004; Johnson et al. 2005), or 'Demand/ Control/Support Questionnaire' (DCS) (Garbarino et al. 2012; Sanne et al. 2005).

Fifth, studies that aim to investigate physiological complaints and ailments are encouraged to consider the 'Psychosomatic Complaints Scale of Stress', PSCSS (Greller and Parsons 1988, 1992), 'Lipp Stress Symptom Inventory', (L)SSI (Lipp 2009), or 'Medical Outcomes Study Short-Form Health Survey' (MOSSF36) (Iwasaki et al. 2002).

Sixth, generic and police-specific measures recommended to address work distress and/or strain include 'Brief Symptom Inventory' (BSI) (Derogatis and Melisaratos 1983; Mearns and Mauch 1998), 'General Health Questions' (GHQ12/GHQ28) (Brown et al. 1999), 'Mental Health Professional Stress Scale' (MHPSS) (Cushway and Tyler 1996), or 'Kessler Psychological Distress Scale' (K10/KPDS) (Noblet et al. 2009). If choosing police-specific measurements, we recommend 'Police Work Experience Survey' (PWES) (Bishopp et al. 2018) or the 'Work Environment Inventory' (WEI) (Liberman et al. 2002).

Seventh, suggested operational and organisational stress measurements include 'Police Stress QuestionnaireOperational/Police Stress Questionnaire-Organizational', 'PSQ-Op/PSQ-Org' (McCreary et al. 2017; McCreary and Thompson 2006), 'Police stressors and felt stress inventory' (Brown and Campbell 1990), or 'Police Stress Questionnaire' PSQ (Biggam et al. 1997). These items all measure demands and stressors in the police setting. Relevant measures for studies concerning acute stress among police include 'Chronic and Episodic Stress' (Anson and Bloom 1988), 'Stress Appraisal Measure' SAM (Peacock and Wong 1990), 'Emotional Stress Reaction Questionnaire' ESRQ (Larsson et al. 1988), and the 'Acute Stress Response Scale' (ASRS) (Yang et al. 2011).

\section{Implications}

The main results from this scoping review show that there are a wide variety of existing measures applied to investigate stress among police. Most measures work well in the context in which they are used. It is recommended that measures be chosen based on the theoretical assumptions concerning stress as a phenomenon in the study in question as well as on the extent to which such measures suit the context and aims of the planned study. It is also advised that measures be chosen to develop both generic and police-specific knowledge. As there seems to be an emphasis on patrolling police work stressors in the context of police-specific stress measures, these stressors may profit from more alignment with the status of (on and offline) police work from several parts of the world to better measure the status quo. More research into stress among police, regardless of police employees' educational background, is also encouraged. More focus should also be devoted to the measurement of stress and wellbeing. Well-chosen measurements can improve the study of stress among police and thereby improve police work and the effect of policing on the societies that police protect and serve.

\section{Appendix}

Appendix search strategies

Ovid MEDLINE(R) ALL 1946 to June 21, 2019

Embase Classic + Embase (Ovid) 1947 to 2019 June

21

PsycINFO (Ovid) 1806 to June Week 32019

AMED (Allied and Complementary Medicine) (Ovid) 1985 to June 2019

Date searched: 23 June, 2019.

\begin{tabular}{|c|c|c|}
\hline 1 & Police/ use medall & 4821 \\
\hline 2 & exp police/ use emczd & 12,188 \\
\hline 3 & Police Personnel/ use psyh & 8880 \\
\hline 4 & $\begin{array}{l}\text { (police* or policing or (law } \\
\text { enforcement adj3 (person- } \\
\text { nel or officer* or official* } \\
\text { or employ* or agen*))). } \\
\text { ti,ab,tw,kw,kf,et }\end{array}$ & 62,454 \\
\hline 5 & or/1-4 & 66,418 \\
\hline 6 & $\begin{array}{l}\text { (exp Occupational Stress/ } \\
\text { or Workload/) use medall }\end{array}$ & 29,960 \\
\hline 7 & $\begin{array}{l}\text { (exp job stress/ or work- } \\
\text { load/) use emczd }\end{array}$ & 49,665 \\
\hline 8 & $\begin{array}{l}\text { (exp occupational stress/ or } \\
\text { Work Load/) use psyh }\end{array}$ & 22,791 \\
\hline 9 & $\begin{array}{l}\text { (Stress Psychological/ or } \\
\text { Workload/) use amed }\end{array}$ & 3077 \\
\hline 10 & $\begin{array}{l}\text { (stress* or workload* or } \\
\text { work load* or demand* } \\
\text { or requirement* or strain } \\
\text { or straining or strained or } \\
\text { ((operational or organisa- } \\
\text { tional or organizational) } \\
\text { adj3 (work or job or } \\
\text { task* or duty or duties } \\
\text { or activit* or factor*))). } \\
\text { ti,ab,tw,kw,kf,et }\end{array}$ & $4,146,872$ \\
\hline 11 & or/6-10 & $4,185,494$ \\
\hline 12 & 5 and 11 & 8080 \\
\hline 13 & $\begin{array}{l}\text { limit } 12 \text { to } y r=" 1806- \\
2005 "\end{array}$ & 2853 \\
\hline 14 & $\begin{array}{l}\text { limit } 12 \text { to } y r=" 2006- \\
2019 "\end{array}$ & 5221 \\
\hline 15 & 13 or 14 & 8074 \\
\hline 16 & 12 not 15 & 6 \\
\hline 17 & remove duplicates from 13 & 2092 \\
\hline 18 & remove duplicates from 14 & 3546 \\
\hline 19 & 16 or 17 or 18 & 5644 \\
\hline
\end{tabular}


The Cochrane Library (Wiley)

Date searched: 23 June, 2019.

\begin{tabular}{|c|c|c|}
\hline$\# 1$ & $\begin{array}{l}\text { MeSH descriptor: [Police] } \\
\text { this term only }\end{array}$ & 64 \\
\hline \#2 & $\begin{array}{l}\text { (police* OR policing OR } \\
\text { ((law NEXT enforce- } \\
\text { ment) NEAR/3 (person- } \\
\text { nel OR officer* OR } \\
\text { official* OR employ* OR } \\
\text { agen*))):ti,ab,kw }\end{array}$ & 601 \\
\hline \#3 & \#1 OR \#2 & 601 \\
\hline \#4 & $\begin{array}{l}\text { MeSH descriptor: [Occupa- } \\
\text { tional Stress] explode all } \\
\text { trees }\end{array}$ & 255 \\
\hline \#5 & $\begin{array}{l}\text { MeSH descriptor: [unde- } \\
\text { fined] explode all trees }\end{array}$ & 0 \\
\hline \#6 & $\begin{array}{l}\text { ((stress* OR workload* OR } \\
\text { (work NEXT load*) OR } \\
\text { demand* OR requirement* } \\
\text { OR strain OR straining OR } \\
\text { strained) OR ((operational } \\
\text { OR organisational OR } \\
\text { organizational) NEAR/3 } \\
\text { (work OR job OR task* OR } \\
\text { duty OR duties OR activit* } \\
\text { OR factor*))):ti,ab,kw }\end{array}$ & 95,970 \\
\hline \#7 & \#4 OR \#5 OR \#6 & 96,039 \\
\hline$\# 8$ & \#3 AND \#7 & 114 \\
\hline$\# 9$ & $\begin{array}{l}\text { \#3 AND \#7 in Cochrane } \\
\text { Reviews, Cochrane Pro- } \\
\text { tocols }\end{array}$ & 2 \\
\hline$\# 10$ & \#3 AND \#7 in Trials & 112 \\
\hline
\end{tabular}

CINAHL (EBSCO)

Date searched: 23 June, 2019.

\begin{tabular}{|c|c|c|}
\hline $\mathrm{S} 1$ & MH "Police" & 5,190 \\
\hline $\mathrm{S} 2$ & $\begin{array}{l}\text { (police* OR policing OR (law enforcement N2 (per- } \\
\text { sonnel OR officer* OR official* OR employ* OR } \\
\text { agen*))) }\end{array}$ & 9,432 \\
\hline $\mathrm{S} 3$ & S1 OR S2 & 9,432 \\
\hline S4 & MH "Stress, Occupational + " OR MH "Workload" & 33,093 \\
\hline S5 & $\begin{array}{l}\text { ((stress* OR workload* OR work load* OR demand* } \\
\text { OR requirement* OR strain OR straining OR } \\
\text { strained) OR ((operational OR organisational OR } \\
\text { organizational) N2 (work OR job OR task* OR duty } \\
\text { OR duties OR activit* OR factor*))) }\end{array}$ & 340,123 \\
\hline S6 & S4 OR S5 & 344,473 \\
\hline S7 & S3 AND S6 & 1,032 \\
\hline S8 & S3 AND S6-Exclude MEDLINE records & 544 \\
\hline
\end{tabular}

\section{Academic Search Premier (EBSCO)}

Criminal Justice Abstracts (EBSCO)

International Security \& Counter Terrorism Reference Center (EBSCO)

Date searched: 4 July, 2019.

\begin{tabular}{|c|c|c|}
\hline S1 & $\mathrm{MH}$ «police» & 33,328 \\
\hline $\mathrm{S} 2$ & $\begin{array}{l}\text { (police* OR policing OR (law enforcement N2 } \\
\text { (personnel OR officer* OR official* OR employ* } \\
\text { OR agen*))) }\end{array}$ & 113,237 \\
\hline S3 & S1 OR S2 & 113,237 \\
\hline S4 & MH "Stress, Occupational + " OR MH "Workload" & 6,841 \\
\hline S5 & $\begin{array}{l}\text { ((stress* OR workload* OR work load* OR } \\
\text { demand* OR requirement* OR strain OR strain- } \\
\text { ing OR strained) OR ((operational OR organi- } \\
\text { sational OR organizational) N2 (work OR job } \\
\text { OR task* OR duty OR duties OR activit* OR } \\
\text { factor*))) }\end{array}$ & $1,685,931$ \\
\hline S6 & S4 OR S5 & $1,685,931$ \\
\hline S7 & S3 AND S6 & 8,937 \\
\hline
\end{tabular}

\section{Scopus}

Date searched: 15 July, 2019.

( TITLE-ABS-KEY ( ( police* OR policing OR ( ( law W/0 enforcement) W/2 ( personnel OR officer* OR official* OR employ* OR agen*))))) AND ( TITLE-ABSKEY ( ( ( stress* OR workload* OR ( work W/0 load*) OR demand* OR requirement* OR strain OR straining OR strained) OR ( ( operational OR organisational OR organizational) W/2 ( work OR job OR task* OR duty OR duties OR activit* OR factor*))))).

Number of hits: 8179 .

Acknowledgements The authors thank Anders Tveit Klette (ATK) for his assistance in the literature searching process. We also want to thank all the librarians working at the Norwegian Police University College who have been priceless at helping us finding articles in full text.

Author contribution LR and BB planned the study and decided on search terms. HS and ATK conducted the systematic literature research and composed the appendix. LR, BB, KIF, and BL analysed and interpreted the data. LR was a major contributor in writing the manuscript and preparing figures and tables. All authors read and approved the final manuscript.

Availability of data and materials All data relevant for this study are included in this published article.

\section{Declarations}

Ethics approval Not applicable.

Competing interests The authors declare no competing interests.

Open Access This article is licensed under a Creative Commons Attribution 4.0 International License, which permits use, sharing, adaptation, distribution and reproduction in any medium or format, as long as you give appropriate credit to the original author(s) and the source, provide a link to the Creative Commons licence, and indicate if changes were made. The images or other third party material in this article are included in the article's Creative Commons licence, unless indicated otherwise in a credit line to the material. If material is not included in the article's Creative Commons licence and your intended use is not permitted by statutory regulation or exceeds the permitted use, you will 
need to obtain permission directly from the copyright holder. To view a copy of this licence, visit http://creativecommons.org/licenses/by/4.0/.

\section{References}

Abdollahi KM (2002) Understanding police stress research. J Forensic Psychol Prac 2(2):1-24. https://doi.org/10.1300/J158v02n02_01

Acquadro Maran D, Varetto A, Zedda M, Franscini M (2014) Stress among Italian male and female patrol police officers: a qualiquantitative survey. Policing: An International Journal of Police Strategies \& Management 37(4):875-890. https://doi.org/10. 1108/PIJPSM-05-2014-0056

Adams IT, Mastracci S (2020) Contrasting emotional labor and burnout in civilian and sworn law enforcement personnel. Policing: An International journal. https://doi.org/10.1108/PIJPSM-06-2019-0094

Alderden M, Skogan WG (2014) The place of civilians in policing. Policing: An International Journal of Police Strategies \& Management 37(2):259-284. https://doi.org/10.1108/ PIJPSM-12-2012-0073

Allisey AF, Noblet AJ, Lamontagne AD, Houdmont J (2014) Testing a model of officer intentions to quit: The mediating effects of job stress and job satisfaction. Crim Justice Behav 41(6):751-771. https://doi.org/10.1177/0093854813509987

Anderson GS, Di Nota PM, Metz GAS, Andersen JP (2019) The impact of acute stress physiology on skilled motor performance: implications for policing. Front Psychol. https://doi.org/10.3389/fpsyg. 2019.02501

Andersson T, Tengblad S (2009) When complexity meets culture: new public management and the Swedish police. Qualitative Research in Accounting Management Research Review 6(1/2):41-56. https:// doi.org/10.1108/11766090910940656

Anshel MH (2000) A conceptual model and implications for coping with stressful events in police work. Crim Justice Behav 27(3):375-400. https://doi.org/10.1177/0093854800027003006

Anshel MH, Robertson M, Caputi P (1997) Sources of acute stress and their appraisals and reappraisals among Australian police as a function of previous experience. J Occup Organ Psychol 70(4):337-356. https://doi.org/10.1111/j.2044-8325.1997.tb00653.x

Anson RH, Bloom ME (1988) Police stress in an occupational context. J Police Sci Admin 16(4):229-235. https://psycnet.apa.org/ record/1990-21261-001

Anson RH, Johnson B, Anson NW (1997) Magnitude and source of general and occupation-specific stress among police and correctional officers. J Offender Rehabil 25(1):103-113. https://doi. org/10.1300/J076v25n01_07

Arble E, Daugherty AM, Arnetz BB (2019) Differential effects of physiological arousal following acute stress on police officer performance in a simulated critical incident. Front Psychol. https:// doi.org/10.3389/fpsyg.2019.00759

Arnetz BB, Nevedal DC, Lumley MA, Backman L, Lublin A (2009) Trauma resilience training for police: Psychophysiological and performance effects. J Police Crim Psych 24(1):1-9. https:// doi.org/10.1007/s11896-008-9030-y

Backteman-Erlanson S, Padyab M, Brulin C (2013) Prevalence of burnout and associations with psychosocial work environment, physical strain, and stress of conscience among Swedish female and male police personnel. Police Prac Res: An International Journal 14(6):491-505. https://doi.org/10.1080/15614263.2012.736719

Bakker AB, Schaufeli WB (2008) Positive organizational behavior: engaged employees in flourishing organizations. J Organ Behav 29(2):147-154. https://doi.org/10.1002/job.515

Barone DF, Caddy GR, Katell AD, Roselione FB, Hamilton RA (1988) The work stress inventory: organizational stress and job risk. Educ Psychol Measur 48(1):141-154. https://doi.org/10. $1177 / 001316448804800117$

Bartol CR, Bergen GT, Volckens JS, Knovas KM (1992) Women in small-town policing: Job performance and stress. Crim Justice Behav 19(3):240. https://doi.org/10.1177/0093854892019003003

Beehr TA, Newman JE (1978) Job stress, employee health, and organizational effectiveness: a facet analysis, model, and literature review. Pers Psychol 31(4):665-699. https://doi.org/10.1111/j.1744-6570. 1978.tb02118.x

Berg AM, Hem E, Lau B, Ekeberg O (2006) An exploration of job stress and health in the Norwegian police service: a cross sectional study. J Occup Med Toxicol 1:26. https://doi.org/10. 1186/1745-6673-1-26

Berg AM, Hem E, Lau B, Haseth K, Ekeberg O (2005) Stress in the Norwegian police service. Occup Med 55(2):113-120. https:// doi.org/10.1093/occmed/kqi023

Berg AM, Hem E, Lau B, Loeb M, Ekeberg O (2003) Suicidal ideation and attempts in Norwegian police. Suicide \& LifeThreatening Behavior 33(3):302-312. https://doi.org/10.1521/ suli.33.3.302.23215

Bertilsson J, Niehorster DC, Fredriksson PJ, Dahl M, Simon G, Fredriksson O, Nyström M (2019) Stress levels escalate when repeatedly performing tasks involving threats. Front Psychol. https://doi.org/10.3389/fpsyg.2019.01562

Bhui K, Dinos S, Galant-Miecznikowska M, de Jongh B, Stansfeld S (2016) Perceptions of work stress causes and effective interventions in employees working in public, private and non-governmental organisations: a qualitative study. Bjpsych Bulletin 40:318-325. https://doi. org/10.1192/pb.bp.115.050823

Biggam FH, Power KG, Macdonald RR, Carcary WB, Moodie E (1997) Self-perceived occupational stress and distress in a Scottish police force. Work Stress 11(2):118-133. https://doi. org/10.1080/02678379708256829

Bishopp SA, Boots DP (2014) General strain theory, exposure to violence, and suicide ideation among police officers: a gendered approach. J Crim Just 42(6):538-548. https://doi.org/10.1016/j. jcrimjus.2014.09.007

Bishopp SA, Piquero NL, Worrall JL, Piquero AR (2018) Negative affective responses to stress among urban police officers: a general strain theory approach. Deviant Behav 40(6):635-654. https://doi.org/10. 1080/01639625.2018.1438069

Bishopp SA, Worrall J, Piquero NL (2016) General strain and police misconduct: the role of organizational influence. Policing-an International Journal of Police Strategies \& Management 39(4):635-651. https://doi.org/10.1108/PIJPSM-10-2015-0122

Bitner C (2019) The impact of work stress and lack of harmony on police officer performance. Law Enforcement Executive Forum 19(2):28-40

Bjørgo T, Damen M-L (2020) The making of a police officer: comparative perspectives on police education and recruitment. Routledge

Bliese PD, Edwards JR, Sonnentag S (2017) Stress and well-being at work: a century of empirical trends reflecting theoretical and societal influences. J Appl Psychol 102(3):389-402. https:// doi.org/10.1037/ap10000109

Bonnet-Suard A, Fernandez L, Marpeaux V, Graziani P, Pedinielli J-L, Rouan G (2005) Stress, tobacco smoking and other addictive behaviours in the police force. Alcoologie Et Addictologie 27(2):26S-36S. https://hal.archives-ouvertes.fr/hal-02350649

Bova N, De Jonge J, Guglielmi D (2013) The demand-induced strain compensation questionnaire: A cross-national validation study. Stress Health 31(3):236-244. https://doi.org/10.1002/smi.2550

Bride BE, Robinson MM, Yegidis B, Figley CR (2004) Development and validation of the secondary traumatic stress scale. Res Soc Work Pract 14(1):27-35. https://doi.org/10.1177/ $1049731503254106 h$ 
Brisinda D, Venuti A, Cataldi C, Efremov K, Intorno E, Fenici R (2015) Real-time imaging of stress-induced cardiac autonomic adaptation during realistic force-on-force police scenarios. J Police Crim Psychol 30(2):71-86. https://doi.org/10.1007/ s11896-014-9142-5

Brown GR (2016) The blue line on thin ice: police use of force modifications in the era of cameraphones and YouTube. Br J Criminol 56(2):293-312. https://doi.org/10.1093/bjc/azv052

Brown J, Campbell EA (1994) Stress and policing: sources and strategies. John Wiley and Sons

Brown J, Cooper C, Kirkcaldy B (1996) Occupational stress among senior police officers. Br J Psychol 87:31-41. https://doi.org/ 10.1111/j.2044-8295.1996.tb02575.x

Brown J, Fielding J, Grover J (1999) Distinguishing traumatic, vicarious and routine operational stressor exposure and attendant adverse consequences in a sample of police officers. Work Stress 13(4):312-325. https://doi.org/10.1080/02678379950019770

Brown J, Grover J (1998) The role of moderating variables between stressor exposure and being distressed in a sample of serving police officers. Personality Individ Differ 24(2):181-185. https://doi.org/10.1016/S0191-8869(97)00156-6

Brown JM, Campbell EA (1990) Sources of occupational stress in the police. Work Stress 4(4):305-318. https://doi.org/10.1080/ 02678379008256993

Burke RJ, Shearer J, Deszca E (1984) Correlates of burnout phases among police officers. Group \& Organization Studies 9(4):451466. https://doi.org/10.1177/105960118400900403

Burnett ME, Sheard I, St Clair-Thompson H (2019) The prevalence of compassion fatigue, compassion satisfaction and perceived stress, and their relationships with mental toughness, individual differences and number of self-care actions in a UK police force. An International Journal, Police Pract Res. https://doi.org/10. 1080/15614263.2019.1617144

Can SH, Hendy HM, Karagoz TJ (2015) LEOSS-R: four types of police stressors and negative psychosocial outcomes associated with them. Policing: J Policy Pract 9(4):340-351. https://doi.org/10. 1093/police/pav011

Caplan RD, Cobb S, French JJRP, Van Harrison R, Pinneau JSR (1975) Job demands and workers health: main effects and occupational differences

Carlson DS, Kacmar KM, Williams LJ (2000) Construction and initial validation of multidimensional measure of work-family conflict. J Vocat Behav 56(2):249-276. https://doi.org/10.1006/jvbe.1999.1713

Chang SJ, Koh SB, Kang D, Kim SA, Kang MG, Lee CG, ... Chae CH (2005) Developing an occupational stress scale for Korean employees. Korean J Occup Environ Med 17(4):297-317. https:// doi.org/10.35371/kjoem.2005.17.4.297

Chiorri C, Garbarino S, Bracco F, Magnavita N (2015) Personality traits moderate the effect of workload sources on perceived workload in flying column police officers. Front Psychol 6:1835. https://doi.org/10.3389/fpsyg.2015.01835

Cockcroft T (2020) Police occupational culture. Policy Press

Cohen S, Kamarck T, Mermelstein R (1983) A global measure of perceived stress. J Health Soc Behav 24:385-396. http://www.jstor. com/stable/2136404

Cohen S, Murphy MLM, Prather AA (2019) Ten surprising facts about stressful life events and disease risk. Annu Rev Psychol 70:577597. https://doi.org/10.1146/annurev-psych-010418-102857

Cohen S, Williamson G (1988) Perceived stress in a probability sample of the United States. In S. Spacapan \& O. S. (Eds.), The social psychology of health: Claremont symposium on applied social psychology (pp. 31-67). SAGE

Collins PA, Gibbs AC (2003) Stress in police officers: a study of the origins, prevalence and severity of stress-related symptoms within a county police force. Occupational Medicine (oxford) 53(4):256-264. https://doi.org/10.1093/occmed/kqg061
Coman GJ, Evans BJ, Stanley RO, Burrows GD (1991) Police officers' work environment and perceptions of stress: an Australian police sample. J Police Crim Psychol 7(1):18-23. https://doi.org/10. 1007/BF02806602

Cooper CL, Davidson MJ, Robinson P (1982) Stress in the police service. J Occup Health 24(1):30-36. https://europepmc.org/article/ $\mathrm{med} / 7054479$

Crank JP, Caldero M (1991) The production of occupational stress in medium-sized police agencies: A survey of line officers in eight municipal departments. J Crim Justice 19(4):339-349. https:// doi.org/10.1016/0047-2352(91)90031-P

Crank JP, Regoli R, Hewitt JD, Culbertson RG (1995) Institutional and organizational antecedents of role stress, work alienation and anomie among. Crim Justice Behav 22(2):152. http:// search.ebscohost.com/login.aspx?direct $=$ true $\& \mathrm{db}=\mathrm{cja} \& \mathrm{AN}=$ $9505302494 \&$ site $=$ ehost-live $\&$ scope $=$ site

Craun SW, Bourke ML, Bierie DM, Williams KS (2014) A longitudinal examination of secondary traumatic stress among law enforcement. Victims \& Offenders 9(3):299-316. https://doi.org/10. 1080/15564886.2013.848828

Cullen FT, Lemming T, Link BG, Wozniak JF (1985) The impact of social supports on police stress. Criminol Public Policy 23(3):503522. https://doi.org/10.1111/j.1745-9125.1985.tb00351.x

Cushway D, Tyler PA (1996) Development of a stress scale for mental health professionals. Br J Clin Psychol 35:279-295. https://doi.org/ 10.1111/j.2044-8260.1996.tb01182.x

Danner D (2016) Reliability-the precision of a measurement. GESIS Survey Guidelines. https://doi.org/10.15465/gesis-sg_en_011

de Souza AC, Alexandre NMC, Guirardello EdB (2017) Psychometric properties in instruments evaluation of reliability and validity. Epidemiologia e Serviços De Saúde 26:649-659. https://doi.org/ 10.5123/S1679-49742017000300022

del Carmen A, Taylor Greene H, Nation DD, Osho GS (2007) Minority women in policing in Texas: An attitudinal analysis. Crim Justice Stud 20(3):281-294. https://doi.org/10.1080/14786010701617680

Delprino RP, O'Quin K, Kennedy C (1997) Identification of work and family services for law enforcement personnel. Police Research and Education Project

Demou E, Hale H, Hunt K (2020) Understanding the mental health and wellbeing needs of police officers and staff in Scotland. Police Pract Res 21:702-716. https://doi.org/10.1080/15614263.2020.1772782

Derogatis LR, Melisaratos N (1983) The brief symptom inventory: an introductory report. Psychol Med 13(3):595-605. https://doi.org/ $10.1017 /$ S0033291700048017

DeVellis RF (2017) Scale development: Theory and applications (4 ed.). Sage publications

Dewe P (1991) Primary appraisal, secondary appraisal and coping: their role in stressful work encounters. J Occup Psychol 64:331351. https://doi.org/10.1111/j.2044-8325.1991.tb00564.x

Dewe PJ, Cooper CL (2017) Work stress and coping: forces of change and challenges. SAGE

Dharmangadan B (1988) Stress at work: a comparison of five occupations. Psychol Stud 33(3):162-169. https://psycnet.apa.org/ record/1989-24371-001

Diamantopoulos A, Winklhofer HM (2001) Index construction with formative indicators: an alternative to scale development. J Mark Res 38(2):269-277. https://doi.org/10.1509/jmkr.38.2. 269.18845

Dollard M, Winefield HR, Winefield AH (2003) Occupational stress in the service professions. CRC Press

Dollard MF, Osborne K, Manning I (2013) Organization-environment adaptation: a macro-level shift in modeling work distress and morale. J Organ Behav 34(5):629-647. https://doi.org/10.1002/ job. 1821

Douma NB, Cote C, Lacasse A (2018) Occupational and ergonomic factors associated with low back pain among car-patrol police 
officers: findings from the Quebec serve and protect low back pain study. Clin J Pain 34(10):960-966. https://doi.org/10. 1097/AJP.0000000000000617

Edwards A, Joyner K, Schatschneider C (2021) A simulation study on the performance of different reliability estimation methods. Educational and Psychological Measurement 1-29. https://doi. org/10.1177/0013164421994184

Edwards JA, Webster S, Van Laar D, Easton S (2008) Psychometric analysis of the UK Health and Safety Executive's Management Standards work-related stress Indicator Tool. Work Stress 22(2):96-107. https://doi.org/10.1080/02678370802166599

Ermasova N, Cross AD, Ermasova E (2020) Perceived stress and coping among law enforcement officers: an empirical analysis of patrol versus non-patrol officers. J Police Crim Psychol 35:48-63. https://doi.org/10.1007/s11896-019-09356-z

Evans BJ, Coman GJ (1993) General versus specific measures of occupational stress: an Australian police survey. Stress Medicine 9(1):11-20. https://doi.org/10.1002/smi.2460090105

Evers A, Frese M, Cooper CL (2000) Revisions and further developments of the occupational stress indicator: LISREL results from four Dutch studies. J Occup Organ Psychol 73(2):221240. https://doi.org/10.1348/096317900166994

Faragher EB, Cooper CL, Cartwright S (2004) A shortened stress evaluation tool (ASSET). Stress Health 20:189-201. https:// doi.org/10.1002/smi.1010

Gaines LK, Van Tubergen N (1989) Job stress in police work: an exploratory analysis into structural causes. Am J Crim Justice 13(2):197-214. https://doi.org/10.1007/BF02887509

Galbraith N, Boyda D, McFeeters D, Galbraith V (2021) Patterns of occupational stress in police contact and dispatch personnel: implications for physical and psychological health. Int Arch Occup Environ Health 94(2):231-241. https://doi.org/10.1007/ s00420-020-01562-1

Garbarino S, Magnavita N, Chiorri C, Brisinda D, Cuomo G, Venuti A, Fenici R (2012) Evaluation of operational stress in riot and crowd control police units: a global challenge for prevention and management of police task-related stress. J Police Crim Psychol 27(2):111-122. https://doi.org/10.1007/s11896-012-9104-8

Garbarino S, Magnavita N, Elovainio M, Heponiemi T, Ciprani F, Cuomo G, Bergamaschi A (2011) Police job strain during routine activities and a major event. Occup Med (Lond) 61(6):395-399. https://doi.org/10.1093/occmed/kqr058

Garcia D, Ryberg F, Arnten A-CA, Archer T, Nima AA (2017) Autonomy and responsibility as a dual construct: Swedish police personnel's stress, energy and motivasjon. Int J Police Sci Manag 19(3):195-204. https://doi.org/10.1177/1461355717714002

Garcia L, Nesbary DK, Gu J (2004) Perceptual variations of stressors among police officers during an era of decreasing crime. J Contemp Crim Justice 20(1):33-50. https://doi.org/10.1177/1043986203262300

Gerber M, Kellmann M, Elliot C, Hartmann T, Brand S, Holsboer-Trachsler E, Puhse U (2013) Perceived fitness protects against stress-based mental health impairments among police officers who report good sleep. J Occup Health 55(5):376-384. https://doi.org/10.1539/joh. 13-0030-OA

Gershon RR, Barocas B, Canton AN, Li X, Vlahov D (2009) Mental, physical, and behavioral outcomes associated with perceived work stress in police officers. Crim Justice Behav 36(3):275-289. https://doi.org/10.1177/0093854808330015

Ghazinour M, Padyab M, Hansson J (2021) Police stress in the Swedish Context: development and psychometric properties of the police stress identification questionnaire. Nordic Journal of Studies in Policing 8(01):1-19. https://doi.org/10.18261/ISSN. 2703-7045-2021-01-05

Giessing L, Frenkel MO, Zinner C, Rummel J, Engel FA, Plessner $\mathrm{H}$ (2019) Effects of coping-related traits and psychophysiological stress responses on police recruits' shooting behavior in reality-based scenarios. Front Psychol. https://doi.org/10.3389/ fpsyg.2019.01523

Glasberg A-L, Eriksson S, Dahlqvist V, Lindahl E, Strandberg G, Söderberg A, ... Norberg AJNE (2006) Development and initial validation of the stress of conscience questionnaire. 13(6):633648. https://doi.org/10.1177/0969733006069698

Gomes R, Lopes H, Simaes C, Afonso J (2016) Occupational stress among Portuguese police officers: A qualitative analysis. Av en Psicol Latinoam 34(1):47-65. https://doi.org/10.12804/apl34.1. 2016.04

Gotts G, Cox T (1988) Stress and arousal checklist: a manual for its administration, scoring and implementation. Swinburne Press

Graf FA (1986) The relationship between social support and occupational stress among police officers. J Police Sci Admin 14(3):178186. https://psycnet.apa.org/record/1987-36270-001

Greller M, Parsons CK (1988) Psychosomatic complaints scale of stress: Measure development and psychometric properties. Educ Psychol Measur 48(4):1051-1065. https://doi.org/10.1177/ 0013164488484022

Greller MM, Parsons CK (1992) Feedback and feedback inconsistency as sources of strain and self-evaluation. Human Relations 45(6):601-620. https://doi.org/10.1177/001872679204500604

Gudjonsson GH, Adlam K (1985) Occupational stressors among British police officers. Police J 58(1):73-80. https://doi.org/10.1177/ $0032258 X 8505800110$

Gutshall CL, Hampton DP Jr, Sebetan IM, Stein PC, Broxtermann TJ (2017) The effects of occupational stress on cognitive performance in police officers. Police Pract Res Int J 18(5):463-477. https://doi.org/10.1080/15614263.2017.1288120

Gächter M, Savage D, Torkler B (2011) The relationship between stress, strain and social capital. Policing an International Journal of Police Strategies and Management 34(3):515-540. https://doi. org/10.1108/13639511111157546

Haarr RN, Morash M (1999) Gender, race, and strategies of coping with occupational stress in policing. Justice Syst J 16(2):303332. https://doi.org/10.1080/07418829900094151

Haisch DC, Meyers LS (2004) MMPI-2 assessed post-traumatic stress disorder related to job stress, coping, and personality in police agencies. Stress Health 20(4):223-229. https://doi.org/10.1002/ smi. 1020

Hall GB, Dollard MF, Coward J (2010) Psychosocial safety climate: Development of the PSC-12. Int J Stress Manag 17(4):353. https://doi.org/10.1037/a0021320

Hansson J, Hurtig AK, Lauritz LE, Padyab M (2017) Swedish police officers' job strain, work-related social support and general mental health. J Police Crim Psychol 32(2):128-137. https://doi.org/ 10.1007/s11896-016-9202-0

Hart PM, Wearing AJ, Headey B (1993) Assessing police work experiences: development of the police daily hassles and uplifts scales. J Crim Just 21(6):553-572. https://doi.org/10.1016/00472352(93)90043-M

Hart PM, Wearing AJ, Headey B (1994) Perceived quality of life, personality, and work experiences: construct validation of the police daily hassles and uplifts scales. Crim Justice Behav 21(3):283311. https://doi.org/10.1177/0093854894021003001

Hartley TA, Violanti JM, Sarkisian K, Fekedulegn D, Mnatsakanova A, Andrew ME, Burchfiel CM (2014) Association between police-specific stressors and sleep quality: influence of coping and depressive symptoms. Journal of Law Enforcement Leadership and Ethics 1(1):31-48. https://www.ncbi.nlm.nih. gov/pmc/articles/PMC5418587/

Hesketh I, Cooper C (2017) Measuring the people fleet: general analysis, interventions and needs. Strateg HR Rev 16(1):17-23. https:// doi.org/10.1108/SHR-10-2016-0092

Hesketh I, Cooper C, Ivy J (2019) Leading the asset: resilience training efficacy in UK policing. The Police Journal: Theory, 
Practice and Principles 92(1):56-71. https://doi.org/10.1177/ $0032258 X 18763101$

Hickman MJ, Fricas J, Strom KJ, Pope MW (2011) Mapping police stress. Police Q 14(3):227-250. https://doi.org/10.1177/ 1098611111413991

Hillgren JS, Bond R, Jones S (1976) Primary stressors in police administration and law enforcement. J Police Sci Admin 4(4):445-449

Hobfoll SE (1989) Conservation of resources: an attempt at conceptualizing stress. Am Psychol 44(3):513-524. https://doi.org/10. 1037/0003-066X.44.3.513

Holmes T, Rahe RH (1967) The social readjustment rating scale. J Psychosom Res 11:213-218. https://doi.org/10.1016/0022-3999(67)90010-4

Horowitz M, Wilner N, Alvarez W (1979) Impact of event scale: a measure of subjective stress. Psychosom Med 41(3):209-218. https://doi.org/10.1097/00006842-197905000-00004

Hurrell JJ Jr, Nelson DL, Simmons BL (1998) Measuring job stressors and strains: where we have been, where we are, and where we need to go. J Occup Health Psychol 3(4):368-389. https://doi. org/10.1037//1076-8998.3.4.368

Iwasaki Y, Mannell RC, Smale BJ, Butcher J (2002) A short-term longitudinal analysis of leisure coping used by police and emergency response service workers. J Leis Res 34(3):311-339. https://doi.org/ 10.1080/00222216.2002.11949974

Johnson S, Cooper C, Cartwright S, Donald I, Taylor P, Millet C (2005) The experience of work-related stress across occupations. J Manag Psychol 20(2):178-187. https://doi.org/10.1108/02683940510579803

Juniper B, White N, Bellamy P (2010) A new approach to evaluating the well-being of police. Occup Med 60(7):560-565. https://doi. org/10.1093/occmed/kqq130

Kanner AD, Coyne JC, Schaefer C, Lazarus RS (1981) Comparison of two modes of stress measurement: daily hassles and uplifts versus major life events. J Behav Med 4(1):1-39. https://doi.org/ 10.1007/BF00844845

Karanika-Murray M, Antoniou A, Michaelides G, Cox T (2009) Expanding the methodology of risk assessment for work-related health: incorporating multivariate curvilinear effects in risk estimation. Work Stress 23(2):99-119. https://doi.org/10.1080/ 02678370903068520

Karasek R, Brisson C, Kawakami N, Houtman I, Bongers P, Amick B (1998) The Job Content Questionnaire (JCQ): an instrument for internationally comparative assessments of psychosocial job characteristics. J Occup Health Psychol 3(4):322. https://doi.org/ 10.1037/1076-8998.3.4.322

Keszei AP, Novak M, Streiner DL (2010) Introduction to health measurement scales. J Psychosom Res 68(4):319-323. https://doi.org/10. 1016/j.jpsychores.2010.01.006

Kirkcaldy B, Brown J, Cooper CL (1998) The demographics of occupational stress among police superintendents. J Manag Psychol 13(1):90-101. https://doi.org/10.1108/02683949810369156

Kirkcaldy B, Shephard R (2001) Occupational stress, work satisfaction and health among the helping professions. Eur Rev Appl Psychol 51(4):243-253. https://psycnet.apa.org/record/2002-15100-002

Kohan A, O'Connor BP (2002) Police officer job satisfaction in relation to mood, well-being, and alcohol consumption. J Psychol Interdiscip Appl 136(3):307-318. https://doi.org/10.1080/ 00223980209604158

Kop N, Euwema M, Schaufeli W (1999) Burnout, job stress and violent behaviour among Dutch police officers. Work Stress 13(4):326340. https://doi.org/10.1080/02678379950019789

Korre M, Farioli A, Varvarigou V, Sato S, Kales SN (2014) A survey of stress levels and time spent across law enforcement duties: police chief and officer agreement. Policing: J Policy Pract 8(2):109122. https://doi.org/10.1093/police/pau001

Kroenke K, Spitzer RL, Williams JB (2002) The PHQ-15: Validity of a new measure for evaluating the severity of somatic symptoms. Psychosom Med 64(2), 258-266. https://journals.lww.com/psych
osomaticmedicine/Fulltext/2002/03000/The_PHQ_15__Valid ity_of_a_New_Measure_for.8.aspx

Kroes WH (1976) Society's victim - the policeman: an analysis of job stress in policing. Charles $\mathrm{C}$ Thomas

Kroes WH, Hurrell JJ, Margolis B (1974a) Job stress in police administrators. J Police Sci Admin 2(4):381-387. https://psycnet.apa. org/record/1975-32720-001

Kroes WH, Margolis BL, Hurrell JJ (1974b) Job stress in policemen. J Police Sci Admin 2(2):145-155. https://psycnet.apa.org/record/ 1975-10622-001

Langner TS (1962) A twenty-two item screening score of psychiatric symptoms indicating impairment. Journal of Health and Human Behavior 3(4):269-276. https://www.jstor.org/stable/2948599

Larsen LB, Andersson EE, Tranberg R, Ramstrand N (2018) Multi-site musculoskeletal pain in Swedish police: associations with discomfort from wearing mandatory equipment and prolonged sitting. Int Arch Occup Environ Health 91(4):425-433. https://doi.org/ 10.1007/s00420-018-1292-9

Larsson G, Kempe C, Starrin B (1988) Appraisal and coping processes in acute time-limited stressful situations: a study of police officers. Eur J Pers 2(4):259-276. https://doi.org/10.1002/per.2410020404

Laufersweiler-Dwyer DL, Dwyer RG (2000) Profiling those impacted by organizational stressors at the macro intermediate and micro levels of several police agencies. Justice Professional 12(4):443. https:// doi.org/10.1080/1478601X.2000.9959562

Lawrence RA (1984) Police stress and personality factors: A conceptual model. J Crim Justice 12(3):247-263. https://doi.org/10. 1016/0047-2352(84)90072-2

Lazarus RS (1995) Psychological Stress in the Workplace. In: Crandall R, Perrewé PL (eds) Occupational stress: A handbook. Taylor \& Francis Ltd, pp 3-14

Lee S (2002) A study of Korean police sergeants' stress. Int J Comp Appl Crim Justice 26(1):85-99. https://doi.org/10.1080/01924036. 2002.9678680

Lees T, Elliott JL, Gunning S, Newton PJ, Rai T, Lal S (2019) A systematic review of the current evidence regarding interventions for anxiety, PTSD, sleepiness and fatigue in the law enforcement workplace. Ind Health 57:655-667. https://doi.org/10. 2486/indhealth.2018-0088

Leitner LA, Posner I, Lester D (1983) Stress, mood and job satisfaction in police chiefs. Police Chief 50(1):54-55. <Go to ISI >:// WOS:A1983PZ99800018

Lester D, Gallagher J (1980) Stress in police officers and department store managers. Psychol Rep 46(3):882. https://doi.org/10.2466/ pr0.1980.46.3.882

Lester D, Solis A (1981) Type A personality, stress, and job satisfaction in police officers. Percept Mot Skills 51(3):890. https://doi.org/10. 2466/pms.1980.51.3.890

Liberman AM, Best SR, Metzler TJ, Fagan JA, Weiss DS, Marmar CR (2002) Routine occupational stress and psychological distress in police. Policing: An International Journal of Police Strategies \& Management 25(2):421-439. https://doi.org/10.1108/13639510210429446

Lilly MM, Pole N, Best SR, Metzler T, Marmar CR (2009) Gender and PTSD: What can we learn from female police officers? J Anxiety Disord 23(6):767-774. https://doi.org/10.1016/j.janxdis.2009.02.015

Lipp ME (2009) Stress and quality of life of senior Brazilian police officers. Span J Psychol 12(2):593-603. https://doi.org/10.1017/ S1138741600001967

Lord VB (1996) An impact of community policing: Reported stressors, social support, and strain among police officers in a changing police department. J Crim Justice 24(6):503-522. https://doi.org/ 10.1016/S0047-2352(96)00036-0

Lord VB, Gray DO, Pond SB (1991) The police stress inventory - does it measure stress. J Crim Just 19(2):139-149. https://doi.org/10. 1016/0047-2352(91)90048-Z 
Lovibond PF, Lovibond SH (1995) The structure of negative emotional states: Comparison of the Depression Anxiety Stress Scales (DASS) with the Beck Depression and Anxiety Inventories. Behav Res Ther 33(3):335-343. https://doi.org/10.1016/00057967(94)00075-U

Ma CC, Hartley TA, Sarkisian K, Fekedulegn D, Mnatsakanova A, Owens S, Andrew ME (2019) Influence of work characteristics on the association between police stress and sleep quality. Saf Health Work 10(1):30-38. https://doi.org/10.1016/j.shaw.2018. 07.004

Mackay CJ, Cox T, Burrows GD, Lazzenni T (1978) An inventory for the measurement of self-reported stress and arousal. British Journal of Social and Clinical Psychology 17:283-284. https:// doi.org/10.1111/j.2044-8260.1978.tb00280.x

Malach-Pines A, Keinan G (2005) Stress and burnout: The significant difference. Pers Individ Differ 39(3):625-635. https://doi.org/10. 1016/j.paid.2005.02.009

Marmar CR, McCaslin SE, Metzler TJ, Best S, Weiss DS, Fagan J, Neylan T (2006) Predictors of posttraumatic stress in police and other first responders. Ann N Y Acad Sci 1071:1-18. https://doi. org/10.1196/annals.1364.001

Martelli TA, Waters LK, Martelli J (1989) The police stress survey: reliability and relation to job satisfaction and organizational commitment. Psychol Rep 64(1):267-273. https://doi.org/10.2466/ pr0.1989.64.1.267

McCarty WP, Aldirawi H, Dewald S, Palacios M (2019) Burnout in blue: an analysis of the extent and primary predictors of burnout among law enforcement officers in the United States. Police Q 22(3):278-304. https://doi.org/10.1177/1098611119828038

McCreary DR, Fong I, Groll DL (2017) Measuring policing stress meaningfully: establishing norms and cut-off values for the operational and organizational police stress questionnaires. Police Pract Res Int J 18(6):612-623. https://doi.org/10.1080/15614263.2017.1363965

McCreary DR, Thompson MM (2006) Development of two reliable and valid measures of stressors in policing: the operational and organizational police stress questionnaires. Int J Stress Manag 13(4):494-518. https://doi.org/10.1037/1072-5245.13.4.494

McEwen BS (2012) Brain on stress: how the social environment gets under the skin. Proc Natl Acad Sci U S A 109(Suppl 2):17180 17185. https://doi.org/10.1073/pnas.1121254109

McEwen BS, Sapolsky RM (1995) Stress and cognitive function. Curr Opin Neurobiol 5(2):205-216. https://doi.org/10.1016/09594388(95)80028-X

McNeish D (2018) Thanks coefficient alpha, we'll take it from here. Psychol Methods 23(3):412-433. https://doi.org/10.1037/ met0000144

Mearns J, Mauch TG (1998) Negative mood regulation expectancies predict anger among police officers and buffer the effects of job stress. Journal of Nervous \& Mental Disease 186(2):120-125. https://doi.org/10.1097/00005053-199802000-00008

Moher D, Liberati A, Tetzlaff J, Altman DG, Group TP (2009) Preferred reporting items for systematic reviews and meta-analyses: the PRISMA statement. PLoS Med 6(7). https://doi.org/10.1371/ journal.pmed.1000097

Morash M, Haarr NR (1995) Gender, workplace problems, and stress in policing. Justice Q 12(1):113-140. https://doi.org/10.1080/ 07418829500092591

Morash M, Haarr R, Kwak D-H (2006) Multilevel influences on police stress. J Contemp Crim Justice 22(1):26-43. https://doi. org/10.1177/1043986205285055

Moos RH, Fenn CB, Billings AG (1988) Life stressors and social resources: An integrated assessment approach. Soc Sci Med 27(9):999-1002. https://doi.org/10.1016/0277-9536(88)90291-2

Motowidlo SJ, Packard JS, Manning MR (1986) Occupational stress: Its causes and consequences for job performance. J Appl
Psychol 71(4):618-629. https://doi.org/10.1037/0021-9010. 71.4.618

Munn Z, Peters MDJ, Stern C, Tufanary C, McArthur A, Aromataris E (2018) Systematic review or scoping review? Guidance for authors when choosing between a systematic or a scoping review approach. BMC Med Res Methodol 18(143). https://doi.org/10. 1186/s12874-018-0611-x

Nandini Karunanidhi, S., \& Chitra, T. (2015) Job attitude in relation to perceived occupational stress, interrole-conflict and psychological well being of women police. Indian J Posit Psychol 6(1):19-25. https://www.proquest.com/openview/ ce6822ae775b96a876ea9ece3f98be2a/1?pq-origsite $=$ gscholar\& $\mathrm{cbl}=2032133$

Nathawat SS, Dadarwal M (2014) A study of job stress among Rajasthan police personnel. Indian J Health Well Being 5(4):422-426

Nisar SK, Rasheed MI (2020) Stress and performance: investigating relationship between occupational stress, career satisfaction, and job performance of police employees. J Public Aff 20(1). https://doi.org/10.1002/pa.1986

Noblet A, Maharee-Lawler S, Rodwell J (2012) Using job strain and organizational justice models to predict multiple forms of employee performance behaviours among Australian policing personnel. Int J Hum Resour Man 23(14):3009-3026. https:// doi.org/10.1080/09585192.2012.656989

Noblet AJ, Rodwell JJ, Allisey AF (2009) Police stress: the role of the psychological contract and perceptions of fairness. Policingan International Journal of Police Strategies \& Management 32(4):613-630. https://doi.org/10.1108/13639510911000731

Nunnally JC (1978) Psychometric theory (Vol. 2). McGraw-Hill

Oginska-Bulik N (2005) The role of personal and social resources in preventing adverse health outcomes in employees of uniformed professions. Int J Occup Med Environ Health 18(3):233240. https://psycnet.apa.org/record/2006-02556-001

Ouzzani M, Hammady H, Fedorowicz Z, Elmagarmid A (2016) Rayyan - a web and mobile app for systematic reviews. Syst Rev (5:210). https://doi.org/10.1186/s13643-016-0384-4

Oyefese AO (1989) Occupational stress and attitude to work in the Nigerian Police Force. Indian J Behav 13(2):14-22. http://ovidsp. ovid.com/ovidweb.cgi? $=\mathrm{JS} \& \mathrm{CSC}=\mathrm{Y} \& \mathrm{NEWS}=\mathrm{N} \& \mathrm{PAGE}=$ fulltext \&D=psyc3\&AN=1990-15967-001

Padilla K (2020) Sources and severity of stress in a Southwestern police department. Occup Med 70(2):131-134. https://doi.org/ 10.1093/occmed/kqaa018

Pagon M, Spector PE, Cooper CL, Lobnikar B (2011) Managers in suits and managers in uniforms: sources and outcomes of occupational stress. Int J Police Sci Manag 13(3):211-222. https://doi.org/10. 1350/ijps.2011.13.3.245

Pancheri P, Martini A, Tarsitani L, Rosati MV, Biondi M, Tomei F (2002) Assessment of subjective stress in the municipal police force of the city of Rome. Stress Health 18(3):127-132. https:// doi.org/10.1002/smi.936

Patterson BL (1992) Job Experience and perceives job stress among police, correctional, and probation/parole officers. Crim Justice Behav 19(3):260-285. https://doi.org/10.1177/0093854892019003004

Peacock EJ, Wong PT (1990) The stress appraisal measure (SAM): a multidimensional approach to cognitive appraisal. Stress Medicine 6(3):227-236. https://doi.org/10.1002/smi.2460060308

Pendergrass VE, Ostrove NM (1984) A survey of stress in women in policing. J Police Sci Admin 12(3):303-309. https://psycnet.apa. org/record/1985-10999-001

Peters M, Godfrey C, McInerney P, Munn Z, Tricco A, Khalil H (2020) Scoping reviews. In E. Aromataris \& Z. Munn (Eds.), JBI Manual for Evidence Synthesis, JBI 2020. https://doi.org/10.46658/ JBIMES-20-12 
Peters MDJ, Godfrey CM, Khalil H, McInerney P, Parker D, Soares CB (2015) Guidance for conducting systematic scoping reviews. Int J Evid Based Healthc 13(3):141-146. https://doi.org/10.1097/ XEB.0000000000000050

Peterson RA (1994) A meta-analysis of Cronbach's coefficient alpha. J Consum Res 21(2):381-391. https://doi.org/10.1086/209405

Pienaar J, Rothmann S (2003) Job stress in the South African Police Services. 15th Conference of the South African Institute for Management Scientists, Potchefstroom

Pienaar J, Rothmann S, Van De Vijver FJ (2007) Occupational stress, personality traits, coping strategies, and suicide ideation in the South African police service. Crim Justice Behav 34(2):246-258. https://doi.org/10.1177/0093854806288708

Purba A, Demou E (2019) The relationship between organisational stressors and mental wellbeing within police officers: a systematic review. BMC Public Health 19(1286). https://doi.org/10. 1186/s12889-019-7609-0

Queriós C, Passos F, Bártolo A, Marques AJ, Da Silva CF, Pereira A (2020) Burnout and stress measurement in police officers: literature review and a study with the police stress questionnaire. Front Psychol 11:587. https://doi.org/10.3389/fpsyg.2020.00587

Qureshi H, Lambert EG, Keena LD, Frank J (2016) Exploring the association between organizational structure variables and work on family strain among Indian police officers. Crim Justice Stud 29(3):253271. https://doi.org/10.1080/1478601X.2016.1167054

Ramakrishnan J, Majgi SM, Premarajan KC, Lakshminarayanan S, Thangaraj S, Chinnakali P (2013) High prevalence of cardiovascular risk factors among policemen in Puducherry. South India. J Cardiovasc Dis Res 4(2):112-115. https://www.ncbi.nlm.nih. gov/pmc/articles/PMC3770116/pdf/main.pdf

Ranta RS, Sud A (2008) Management of stress and burnout of police personnel. J Indian Acad Appl Psychol 34(1):29-39. http:// ovidsp.ovid.com/ovidweb.cgi? $\mathrm{T}=\mathrm{JS} \& \mathrm{CSC}=\mathrm{Y} \& \mathrm{NEWS}=\mathrm{N} \&$ $\mathrm{PAGE}=$ fulltext $\& \mathrm{D}=$ psyc6\&AN=2008-00522-003

Rasdi I, Nurulizyati R, Nur Fatihah D (2017) Heat strain and work performance among traffic police officers in Kuala Lumpur. Annals of Tropical Medicine \& Public Health 10(1):65-70. https://doi. org/10.4103/ATMPH.ATMPH_77_17

Raykov T, Marcoulides GA (2019) Thanks coefficient alpha, we still need you! Educ Psychol Measur 79(1):200-210. https://doi.org/ 10.1177/0013164417725127

Regehr C, LeBlanc VR (2017) PTSD, Acute stress, performance and decision-making in emergency service workers. J Am Acad Psychiatry Law 45(2):184-192

Reiser M (1976) Stress, distress, and adaption in police work. Police Chief 43(1):24-27. https://www.ojp.gov/ncjrs/virtual-library/ abstracts/stress-distress-and-adaptation-police-work

Reuss-Ianni E (2011) Two cultures of policing: street cops and management cops. Transaction Publishers. https://doi.org/10.4324/9781351300964

Revicki DA, Whitley TW, Gallery ME (1993) Organizational characteristics, perceived work stress, and depression in emergency medicine residents. Behav Med 19(2):74-81. https://doi.org/10. 1080/08964289.1993.9937568

Ricciardelli R (2018) "Risk It Out, Risk It Out": occupational and organizational stresses in rural policing. Police Q 21(4):415-439. https:// doi.org/10.1177/1098611118772268

Richmond RL, Wodak A, Kehoe L, Heather N (1998) How healthy are the police? A survey of life-style factors. Addiction 93(11):17291737. https://doi.org/10.1046/j.1360-0443.1998.9311172910.x

Robertson CT, Cooper CL, Williams J (1990) The validity of the occupational stress indicator. Work Stress 4(1):29-39. https://doi.org/ $10.1080 / 02678379008256962$

Robinson AM (2018) Let's talk about stress: history of stress research. Rev Gen Psychol. https://doi.org/10.1037/gpr0000137

Robinson HM, Sigman MR, Wilson JP (1997) Duty-related stressors and PTSD symptoms in suburban police officers. Psychological
Reports 81(3):835-845. https://doi.org/10.2466/pr0.1997. 81.3.835

Rodrigues S, Sinval J, Queiros C, Maroco J, Kaiseler M (2019) Transitioning from recruit to officer: an investigation of how stress appraisal and coping influence work engagement. Int J Sel Assess 27:152-168. https://doi.org/10.1111/ijsa.12238

Rose T, Unnithan P (2015) In or out of the group? Police subculture and occupational stress. Policing: An International Journal of Police Strategies \& Management 38(2):279-294. https://doi.org/ 10.1108/PIJPSM-10-2014-0111

Rothmann S, Mostert K, Geldenhuys M (2006) A psychometric evaluation of the job demands resources scale in South Africa. J Ind Psychol 32(4):76-86. https://doi.org/10.4102/sajip.v32i4.239

Roz HB, Raval DT (2017) A study of occupational stress, burnout, presumptive life events, depression and anxiety among Ahmedabad city police officers. Indian Journal of Health and Wellbeing 8(12):1485-1492. http://www.iahrw.com/index.php/home/ journal_detail/19\#list

Sandvik AM, Gjevestad E, Aabrekk E, Øhman P, Kjendlie P-L, Hystad SW, Johnsen BH (2020) Physical fitness and psychological hardiness as predictors of parasympathetic control in response to stress: a Norwegian police simulator training study. J Police Crim Psychol 35(4):504-517. https://doi.org/10.1007/ s11896-019-09323-8

Sanne B, Torp S, Mykletun A, Dahl AA (2005) The Swedish Demand-Control-Support Questionnaire (DCSQ): factor structure, item analyses, and internal consistency in a large population. Scand J Public Health 33(3):166-174. https://doi.org/10. 1080/14034940410019217

Saunders J, Kotzias V, Ramchand R (2019) Contemporary police stress: the impact of the evolving socio-political context. Criminology, Criminal Justice, Law \& Society 20(1):35-52. https:// doi.org/10.21202/1993-047X.13.2019.3.1430-1449

Savery LK, Soutar GN, Weaver JR (1993) Stress and the police officer: some West Australian evidence. The Police Journal 66(3):277290. https://doi.org/10.1177/0032258X9306600307

Schardt C, Adams MB, Owens T, Keitz S, Fontelo PJ (2007) Utilization of the PICO framework to improve searching PubMed for clinical questions. BMC Med Inform Decis Mak 7(1):1-6. https://doi. org/10.1186/1472-6947-7-16

Scott YM (2004) Stress among rural and small-town patrol officers: A survey of Pennsylvania municipal agencies. Police Q 7(2):237260. https://doi.org/10.1177/1098611103258958

Sewell JD (1980) The development of a critical life events scale for law enforcement. The Florida State University. https://www.elibrary. $\mathrm{ru} / \mathrm{item}$.asp? $\mathrm{id}=7318732$

Sewell JD (1983) The development of a critical life event scale for law enforcement. J Police Sci Admin 11(1):109-116. https://www. ojp.gov/ncjrs/virtual-library/abstracts/development-critical-lifeevents-scale-law-enforcement

Shane JM (2013) Daily work experiences and police performance. Police Pract Res Int J 14(1):17-34

Shane JM (2020) Stress inside police departments: how the organization creates stress and performance in police officers. Routledge

Shiozaki M, Miyai N, Morioka I, Utsumi M, Hattori S, Koike H, ... Miyashita $\mathrm{K}$ (2017) Job stress and behavioral characteristics in relation to coronary heart disease risk among Japanese police officers. Ind Health 55(4):369-380. https://doi.org/10.2486/indhealth.2016-0179

Simons Y, Barone DF (1994) The relationship of work stressors and emotional support to strain in police officers. Int J Stress Manag 1(3):223-234. https://doi.org/10.1007/BF01857989

Singh S, Kar SK (2015) Sources of occupational stress in the police personnel of North India: An exploratory study. Indian J Occup Environ Med 19(1):56-60. http://www.ijoem.com/article.asp? issn $=0019-5278$; year $=2015 ;$ volume $=19 ;$ issue $=1 ;$ spage $=56$; epage $=60 ;$ aulast $=$ Singh 
Siu OlJAP (2002) Occupational stressors and well-being among Chinese employees: the role of organisational commitment. Applied Psychology: an International Review 51(4):527-544. https://doi. org/10.1111/1464-0597.t01-1-00106

Slate RN, Johnson WW, Colbert SS (2007) Police stress: a structural model. J Police Crim Psychol 22(2):102-112. https://doi.org/10. 1007/s11896-007-9012-5

Spielberger C, Reheiser EC (1994) The job stress survey: measuring gender differences in occupation stress. J Soc Behav Pers 9(2):199-218

Spielberger CD, Gorsuch RL, Lushene RE (1970) Manual for the statetrait anxiety inventory. CA, Consulting Psychologists Press, Palo Alto

Spielberger CD, Westberry LG, Grier KS, Greenfield G (1981) Police stress survey - sources of stress in law enforcement (Monograph, Issue. http://www.ncjrs.gov/App/publications/abstract.aspx?ID= 80993

Stephens C, Long N (1998) Posttraumatic stress disorder in the New Zealand police: the moderating role of social support following traumatic stress. Anxiety Stress Coping 12(3):247-164. https:// doi.org/10.1080/10615809908250477

Stinchcomb JB (2004) Searching for Stress in All the Wrong Places: Combating Chronic Organizational Stressors in Policing. Police Pract Res 5(3):259-277. https://doi.org/10.1080/ 156142604200227594

Strahler J, Ziegert T (2015) Psychobiological stress response to a simulated school shooting in police officers. Psychoneuroendocrinology 51:80-91. https://doi.org/10.1016/j.psyneuen.2014.09.016

Stratton JG (1978) Police Stress - Overview Police Chief 45(4):58-62

Symonds M (1970) Emotional hazards of police work. Am J Psychoanal 30(2):155-160. https://doi.org/10.1007/BF01874038

Symonds M (1972) Policemen and policework: a psychodynamic understanding. Am J Psychoanal 32(2):163-169. https://doi.org/ 10.1007/BF01872512

Talavera-Velasco B, Luceno-Moreno L, Martin-Garcia J, GarciaAlbuerne Y (2018) Psychosocial risk factors, burnout and hardy personality as variables associated with mental health in police officers. Front Psychol 9:1478. https://doi.org/10.3389/fpsyg. 2018.01478

Tang CSK, Lau BHB (1996) Gender role stress and burnout in Chinese human service professionals in Hong Kong. Anxiety Stress Coping 9(3):217-227. https://doi.org/10.1080/10615809608249403

Taverniers J, Smeets T, Van Ruysseveldt J, Syroit J, von Grumbkow J (2011) The risk of being shot at: stress, cortisol secretion, and their impact on memory and perceived learning during realitybased practice for armed officers. Int J Stress Manag 18(2):113132. https://doi.org/10.1037/a0023742

Taylor Greene H, del Carmen A (2002) Female police officers in Texas - Perceptions of colleagues and stress. Policing: An International Journal of Police Strategies and Management 25(2):385-398. https://doi.org/10.1108/13639510210429428

Thompson BM, Kirk A, Brown D (2006) Sources of stress in policewomen: A three-factor model. Int J Stress Manag 13(3):309-328. https://doi.org/10.1037/1072-5245.13.3.309

Tricco AC, Lillie E, Zarin W, O’Brien KK, Colquhoun H, Levac D, Hempel S (2018) PRISMA extension for scoping reviews (PRISMA-ScR): checklist and explanation. Ann Intern Med 169(7):467-473. https://doi.org/10.7326/M18-0850

Trizano-Hermosilla I, Alvarado JM (2016) Best alternatives to Cronbach's alpha reliability in realistic conditions: congeneric and asymmetrical measurements. Front Psychol 7:769. https://doi. org/10.3389/fpsyg.2016.00769

Tuckey MR, Chrisopoulos S, Dollard MF (2012) Job demands, resource deficiencies, and workplace harassment: Evidence for micro-level effects. Int J Stress Manag 19(4):292-310. https:// doi.org/10.1037/a0030317
Tundwal S, Behmani RK (2016) Relationship between organisational role stress and life satisfaction among female police personnel. Indian Journal of Health \& Wellbeing 7(2):225-228. https:// web.s.ebscohost.com/ehost/detail/detail?vid $=0 \& \operatorname{sid}=8 \mathrm{~d} 4 \mathrm{a} 5 \mathrm{c} 06$ 717e-4328-a75b-07cd2d0003ea\%40redis\&bdata=JnNpdGU9ZW hvc3QtbGl2ZSZzY29wZT1zaXRl\#AN=115097102\&db=aph

Tyagi A, Dhar RL (2014) Factors affecting health of the police officials: Mediating role of job stress. Policing: An International Journal of Police Strategies \& Management 37(3):649-664. https://doi. org/10.1108/PIJPSM-12-2013-0128

Van den Broeck A, De Cuyper N, De Witte H, Vansteenkiste M (2010) Not all job demands are equal: Differentiating job hindrances and job challenges in the Job Demands-Resources model. Eur J Work Organ Psychol 19(6):735-759. https://doi.org/10.1080/ 13594320903223839

Van Hasselt VB, Sheehan DC, Malcolm AS, Sellers AH, Baker MT, Couwels J (2008) The Law Enforcement Officer Stress Survey (LEOSS): evaluation of psychometric properties. Behav Modif 32(1):133-151. https://doi.org/10.1177/0145445507308571

Van Hasselt VB, Sheehan DC, Sellers AH, Baker MT, Feiner CA (2003) A behavioral-analytic model for assessing stress in police officers: phase I. Development of the Law Enforcement Officer Stress Survey (LEOSS). International Journal of Emergency Mental Health and Human Resilience 5(2):77-84

Van Patten IT, Burke TW (2001) Critical incident stress and the child homicide investigator. Homicide Studies: an Interdisciplinary \& International Journal 5(2):131-152. https://doi.org/10.1177/ 1088767901005002003

van Woerkom M, Bakker AB, Leiter MP (2021) Positive psychology interventions in organizations. J Occup Organ Psychol 1-9. https://doi.org/10.1111/joop.12350

Violanti J, Fekedulegn D, Charles L, Andrew M, Hartley T, Mnatsakanova A, Burchfiel C (2009) Suicide in police work: exploring potential contributing influences. Am J Crim Justice 34(1):41-53. https://doi.org/10.1007/s12103-008-9049-8

Violanti JM, Charles LE, McCanlies E, Hartley TA, Baughman P, Andrew ME, Burchfiel CM (2017) Police stressors and health: a state-of-the-art review. Policing 40(4):642-656. https://doi.org/ 10.1108/PIJPSM-06-2016-0097

Violanti JM, Fekedulegn D, Hartley TA, Andrew ME, Gu JK, Burchfiel CM (2013) Life expectancy in police officers: a comparison with the U.S. general population. International Journal of Emergency Mental Health and Human Resilience 15(4):217-228. https:// www.ncbi.nlm.nih.gov/pmc/articles/PMC4734369/

Violanti JM, Ma CC, Gu JK, Fekedulegn D, Mnatsakanova A, Andrew ME (2018) Social avoidance in policing: associations with cardiovascular disease and the role of social support. Policing: An International journal 41(5):539-549. https://doi.org/10.1108/ PIJPSM-02-2017-0017

Violanti JM, Ma CC, Mnatsakanova A, Fekedulegn D, Hartley TA, Gu JK, Andrew ME (2018b) Associations between police work stressors and posttraumatic stress disorder symptoms: examining the moderating effects of coping. J Police Crim Psychol 33(3):271-282. https://doi.org/10.1007/s11896-018-9276-y

Violanti JM, Mnatsakanova A, Andrew ME (2013b) Suicidal ideation in police officers: exploring an additional measure. Suicidology Online 4:33-41

Violanti JM, Owens SL, McCanlies E, Fekedulegn D, Andrew ME (2019) Law enforcement suicide: a review. Policing-an International Journal of Police Strategies \& Management 42(2):141164. https://doi.org/10.1108/pijpsm-05-2017-0061

Wang Y, Zheng L, Hu T, Zheng Q (2014) Stress, burnout, and job satisfaction: case of police force in China. Public Personnel Management 43(3):325-339. https://doi.org/10.1177/0091026014535179

Watson D, Clark LA, Tellegen A (1988) Development and validation of brief measures of positive and negative affect: the PANAS 
scales. J Pers Soc Psychol 54(6):1063. https://doi.org/10.1037/ 0022-3514.54.6.1063

Webb SD, Smith DL (1980) Police stress: a conceptual overview. J Crim Just 8(4):251-257. https://doi.org/10.1016/0047-2352(80) 90005-7

Webster JH (2013) Police officer perceptions of occupational stress: the state of the art. Policing-an International Journal of Police Strategies \& Management 36(3):636-652. https://doi.org/10. 1108/PIJPSM-03-2013-0021

Webster JH (2014) Perceived stress among police officers: an integrative model of stress and coping. Policing-an International Journal of Police Strategies \& Management 37(4):839-857. https://doi. org/10.1108/PIJPSM-06-2014-0064

Weiss DS, Brunet A, Best SR, Metzler TJ, Liberman A, Pole N, Marmar CR (2010) Frequency and severity approaches to indexing exposure to trauma: the Critical Incident History Questionnaire for police officers. J Trauma Stress 23(6):734-743. https://doi.org/10.1002/ jts.20576

Weiss DS, Marmar CR, Metzler TJ, Ronfeldt HM (1995) Predicting symptomatic distress in emergency services personnel. Journal of Consulting Clinical Psychology 63(3):361-368. https://doi. org/10.1037/0022-006X.63.3.361

Wexler JG, Logan DD (1983) Sources of stress among women police officers. J Police Sci Admin 11(1):46-53. https://www.ojp.gov/ ncjrs/virtual-library/abstracts/sources-stress-among-womenpolice-officers

White JW, Lawrence P, Biggerstaff C, Grubb TD (1985) Factors of stress among police officers. Crim Justice Behav 12(1):111-128. https://doi.org/10.1177/0093854885012001008

White S, Marino KE (1983) Job attitudes and police stress: An exploratory study of causation. J Police Sci Admin 11(3):264274. https://www.ojp.gov/ncjrs/virtual-library/abstracts/jobattitudes-and-police-stress-exploratory-study-causation

Williams S, Cooper CL (1998) Measuring occupational stress: Development of the pressure management indicator. J Occup Health Psychol 3(4):306-321. https://doi.org/10.1037/1076-8998.3.4.306

Wilson JQ (1978) Varieties of police behavior: the management of law and order in eight communities, with a new preface by the author. Harvard University Press
Winwood PC, Peters R, Peters M, Dollard M (2012) Further validation of the psychological injury risk indicator scale. J Occup Environ Med 54(4):478-484. https://doi.org/10.1097/JOM.0b013e3182479f77

Winwood PC, Tuckey MR, Peters R, Dollard MFJJoo, medicine e (2009) Identification and measurement of work-related psychological injury: piloting the psychological injury risk indicator among frontline police. J Occup Environ Med 51(9):1057-1065. https://doi.org/10.1097/JOM.0b013e3181b2f3d8

Yamauchi T, Yoshikawa T, Sasaki T, Matsumoto S, Takahashi M, Suka M, Yanagisawa H (2018) Cerebrovascular/cardiovascular diseases and mental disorders due to overwork and workrelated stress among local public employees in Japan. Ind Health 56(1):85-91. https://doi.org/10.2486/indhealth.2017-0131

Yang Y, Tang J, Jiang Y, Liu X, Sun Y, Zhu X, Miao D (2011) Development of the acute stress response scale. SOCIAL BEHAVIOR AND PERSONALITY 39(5):713-720. https://doi.org/10.2224/ sbp.2011.39.5.713

Yaribeygi H, Panahi Y, Sahraei H, Johnston TP, Sahebkar A (2017) The impact of stress on body function: a review. EXCLI Journal 16:1057-1072. https://doi.org/10.17179/excli2017-480

Young DR (1994) Can cardiorespiratory fitness moderate the negative effects of stress on coronary artery disease risk factors? J Psychosom Res 38(5):451-459. https://doi.org/10.1016/0022-3999(94)90106-6

Yu H, Liu J-C, Fan Y-J, Li C, Zhang L-X, Chen X, ... Tang N-J (2016) Association between occupational stressors and type 2 diabetes among Chinese police officers: a 4-year follow-up study in Tianjin, China. Int Arch Occup Environ Health 89(2):277-288. https://doi.org/10.1007/s00420-015-1071-9

Zefferino R, Facciorusso A, Lasalvia M, Narciso M, Nuzzaco A, Lucchini R, L'Abbate N (2006) Salivary markers of work stress in an emergency team of urban police (1 degree step). G Ital Med Lav Erg 28(4):472-477. https://citeseerx.ist.psu.edu/viewdoc/download? doi $=10.1 \cdot 1.528 .1072 \&$ rep $=$ rep $1 \&$ type $=$ pdf

Zimmerman FH (2012) Cardiovascular disease and risk factors in law enforcement personnel: a comprehensive review. Cardiol Rev 20(4):159-166. https://doi.org/10.1097/CRD.0b013e318248d631

Publisher's Note Springer Nature remains neutral with regard to jurisdictional claims in published maps and institutional affiliations. 